\title{
Research Article \\ Criteria of Wiener Type for Minimally Thin Sets and Rarefied Sets Associated with the Stationary Schrödinger Operator in a Cone
}

\author{
Pinhong Long, Zhiqiang Gao, and Guantie Deng \\ Laboratory of Mathematics and Complex Systems, School of Mathematical Science, \\ Beijing Normal University, Beijing 100875, China \\ Correspondence should be addressed to Pinhong Long, hnlphlch10601@sina.com and Guantie \\ Deng, denggt@bnu.edu.cn
}

Received 8 April 2012; Accepted 18 June 2012

Academic Editor: Ondřej Došlý

Copyright (c) 2012 Pinhong Long et al. This is an open access article distributed under the Creative Commons Attribution License, which permits unrestricted use, distribution, and reproduction in any medium, provided the original work is properly cited.

\begin{abstract}
We give some criteria for $a$-minimally thin sets and $a$-rarefied sets associated with the stationary Schrödinger operator at a fixed Martin boundary point or $\infty$ with respect to a cone. Moreover, we show that a positive superfunction on a cone behaves regularly outside an $a$-rarefied set. Finally we illustrate the relation between the $a$-minimally thin set and the $a$-rarefied set in a cone.
\end{abstract}

\section{Introduction}

This paper is concerned with some properties for the generalized subharmonic functions associated with the stationary Schrödinger operator. More precisely the minimally thin sets and rarefied sets about these generalized subharmonic functions will be studied. The research on minimal thinness has been exploited a little and attracted many mathematicians. In 1949 Lelong-Ferrand [1] started the study of the thinness at boundary points for the subharmonic functions on the half-space. Then in 1957 Naïm [2] gave some criteria for minimally thin sets at a fixed boundary point with respect to half-space (see [3] for a survey of the results in [1, 2]). In 1980 Essén and Jackson [4] gave the criteria for minimally thin sets at $\infty$ with respect to half-space, and furthermore they introduced rarefied sets at $\infty$ with respect to half-space, which is more refined than minimally thin set. Later Miyamoto and Yoshida [5] extended these results of Essén and Jackson from half-space to a cone. In this paper, we will deal with the corresponding questions for the generalized subharmonic functions associated with the stationary Schrödinger operator. 
To state our results, we will need some notations and preliminary results. As usual, denote by $\mathbf{R}^{n}(n \geq 2)$ the $n$-dimensional Euclidean space. For an open subset set $\mathbf{S} \subset \mathbf{R}^{n}$, denote its boundary by $\partial \mathbf{S}$ and its closure by $\overline{\mathbf{S}}$. Let $P=\left(X, x_{n}\right)$, where $X=\left(x_{1}, x_{2}, \ldots, x_{n-1}\right)$, and let $|P|$ be the Euclidean norm of $P$ and $|P-Q|$ the Euclidean distance of two points $P$ and $Q$ in $\mathbf{R}^{n}$. The unit sphere and the upper half unit sphere are denoted by $\mathbf{S}^{n-1}$ and $\mathbf{S}_{+}^{n-1}$, respectively. For $P \in \mathbf{R}^{n}$ and $r>0$, let $B(P, r)$ be the open ball of radius $r$ centered at $P$ in $\mathbf{R}^{n}$, then $S_{r}=\partial B(O, r)$. Furthermore, denote by $d S_{r}$ the $(n-1)$-dimensional volume elements induced by the Euclidean metric on $S_{r}$.

For $P=\left(X, x_{n}\right) \in \mathbf{R}^{n}$, it can be reexpressed in spherical coordinates $(r, \Theta), \Theta=$ $\left(\theta_{1}, \theta_{2}, \ldots, \theta_{n}\right)$ via the following transforms:

$$
x_{1}=r \prod_{j=1}^{n-1} \sin \theta_{j} \quad(n \geq 2), x_{n}=r \cos \theta_{1}
$$

and if $n \geq 3$,

$$
x_{n-k+1}=r \cos \theta_{k} \prod_{j=1}^{k-1} \sin \theta_{j} \quad(2 \leq k \leq n-1)
$$

where $0 \leq r<\infty, 0 \leq \theta_{j} \leq \pi(1 \leq j \leq n-2 ; n \geq 3)$ and $-\pi / 2 \leq \theta_{n-1} \leq(3 \pi / 2)(n \geq 2)$.

Relative to the system of spherical coordinates, the Laplace operator $\Delta$ may be written as

$$
\Delta=\frac{n-1}{r} \frac{\partial}{\partial r}+\frac{\partial^{2}}{\partial r^{2}}+\frac{\Delta^{*}}{r^{2}}
$$

where the explicit form of the Beltrami operator $\Delta^{*}$ is given by Azarin (see [6]).

Let $D$ be an arbitrary domain in $\mathbf{R}^{n}$, and $\mathscr{A}_{D}$ denotes the class of nonnegative radial potentials $a(P)$ (i.e., $0 \leq a(P)=a(r)$ for $P=(r, \Theta) \in D$ ) such that $a \in L_{\text {loc }}^{b}(D)$ with some $b>n / 2$ if $n \geq 4$ and with $b=2$ if $n=2$ or $n=3$. $a(\cdot)$ by

For the identical operator $I$, define the stationary Schrödinger operator with a potential

$$
\mathcal{L}_{a}=-\Delta+a(\cdot) I
$$

If $a \in \mathcal{A}_{D}$, then $\mathcal{L}_{a}$ can be extended in the usual way from the space $C_{0}^{\infty}(D)$ to an essentially self-adjoint operator on $L^{2}(D)$ (see [7, Chapter 13] for more details). Furthermore $\mathcal{L}_{a}$ has a Green $a$-function $G_{D}^{a}(\cdot, \cdot)$. Here $G_{D}^{a}(\cdot, \cdot)$ is positive on $D$, and its inner normal derivative $\partial G_{D}^{a}(\cdot, Q) / \partial n_{Q}$ is nonnegative, where $\partial / \partial n_{Q}$ denotes the differentiation at $Q$ along the inward normal into $D$. We write this derivative by $P I_{D}^{a}(\cdot, \cdot)$, which is called the Poisson $a$ kernel with respect to $D$. Denote by $G_{D}^{0}(\cdot, \cdot)$ the Green function of Laplacian. It is well known that

$$
G_{D}^{a}(\cdot, \cdot) \leq G_{D}^{0}(\cdot, \cdot)
$$


for any potential $a(\cdot) \geq 0$. The "inverse" inequality in some sense is much more elaborate. When $D$ is a bounded domain in $\mathbf{R}^{n}$, Cranston (see [8], the case $n=2$ is implicitly contained in [9]) have proved that

$$
G_{D}^{a}(\cdot, \cdot) \geq M(D) G_{D}^{0}(\cdot, \cdot)
$$

where $M(D)=M(D, a)$ is a positive constant and independent of points in $D$. If $a=0$, then obviously $M(D) \equiv 1$.

Suppose that a function $u \not \equiv-\infty$ is upper semicontinuous in $D$. We call $u \in[-\infty,+\infty)$ a subfunction for the Schrödinger operator $\mathcal{L}_{a}$ if the generalized mean-value inequality

$$
u(P) \leq \int_{S(P, \rho)} u(Q) \frac{\partial G_{B(P, \rho)}^{a}(P, Q)}{\partial n_{Q}} d \sigma(Q)
$$

is satisfied at each point $P \in D$ with $0<\rho<\inf _{Q \in \partial D}|P-Q|$, where $S(P, \rho)=\partial B(P, \rho)$, $G_{B(P, \rho)}^{a}(\cdot, \cdot)$ is the Green $a$-function of $\perp_{a}$ in $B(P, r)$, and $d \sigma(\cdot)$ the surface area element on $S(P, \rho)$ (see [10]).

Denote by $\operatorname{SbH}(a, D)$ the class of subfunctions in $D$. We call $u$ a superfunction associated with $\mathcal{L}_{a}$ if $-u \in \operatorname{SbH}(a, D)$, and denote by $\operatorname{SpH}(a, D)$ the class of superfunctions. If a function $u$ on $D$ is both subfunction and superfunction, then it is called an $a$-harmonic function associated with the operator $\mathcal{L}_{a}$. The class of $a$-harmonic functions is denoted by $H(a, D)$, and it is obviously $\operatorname{SbH}(a, D) \cap \operatorname{SpH}(a, D)$. Here we follow the terminology from Levin and Kheyfits (see [11-13]).

For simplicity, the point $(1, \Theta)$ on $\mathbf{S}^{n-1}$ and the set $\{\Theta ;(1, \Theta) \in \Omega\}$ for a set $\Omega \subset \mathbf{S}^{n-1}$ are often identified with $\Theta$ and $\Omega$, respectively. For $\Xi \subset \mathbf{R}_{+}$and $\Omega \subset \mathbf{S}^{n-1}$, the set $\{(r, \Theta) \in$ $\left.\mathbf{R}^{n} ; r \in \Xi,(1, \Theta) \in \Omega\right\}$ in $\mathbf{R}^{n}$ is simply denoted by $\Xi \times \Omega$. In particular, the half space $\left\{\left(X, x_{n}\right) \in\right.$ $\left.\mathbf{R}^{n} ; x_{n}>0\right\}=\mathbf{R}_{+} \times \mathbf{S}_{+}^{n-1}$ will be denoted by $\mathbf{T}_{n}$. We denote by $C_{n}(\Omega)$ the set $\mathbf{R}_{+} \times \Omega$ in $\mathbf{R}^{n}$ with the domain $\Omega \subset \mathbf{S}^{n-1}$ and call it a cone. For an interval $I \subset \mathbf{R}_{+}$and $\Omega \subset \mathbf{S}^{n-1}$, write $C_{n}(\Omega ; I)=I \times \Omega$, $S_{n}(\Omega ; I)=I \times \partial \Omega$, and $C_{n}(\Omega ; r)=C_{n}(\Omega) \cap S_{r}$. By $S_{n}(\Omega)$ we denote $S_{n}(\Omega ;(0,+\infty))$, which is $\partial C_{n}(\Omega)-\{O\}$. From now on, we always assume $D=C_{n}(\Omega)$ and write $G_{\Omega}^{a}(\cdot, \cdot)$ instead of $G_{C_{n}(\Omega)}^{a}(\cdot, \cdot)$.

Let $\Omega$ be a domain on $\mathbf{S}^{n-1}$ with smooth boundary. Suppose that $\tau$ is the least positive eigenvalue for $-\Delta^{*}$ on $\Omega$ and the normalized positive eigenfunction $\varphi(\Theta)$ corresponding to $\tau$ satisfies $\int_{\Omega} \varphi^{2}(\Theta) d S_{1}=1$. Then

$$
\begin{gathered}
\left(\Delta^{*}+\tau\right) \varphi(\Theta)=0 \text { on } \Omega, \\
\varphi(\Theta)=0 \text { on } \partial \Omega
\end{gathered}
$$

(see [14, page 41]). In order to ensure the existence of $\tau$ and $\varphi(\Theta)$, we pose the assumption on $\Omega$ : if $n \geq 3$, then $\Omega$ is a $C^{2, \alpha}$-domain $(0<\alpha<1)$ on $S^{n-1}$ surrounded by a finite number of mutually disjoint closed hypersurfaces (see e.g., [15, pages 88-89] for the definition of $C^{2, \alpha}$ domain).

Let $B_{D}$ be the class of the potential $a \in \mathscr{A}_{D}$ such that

$$
\lim _{r \rightarrow \infty} r^{2} a(r)=\kappa_{0} \in[0, \infty), \quad r^{-1}\left|r^{2} a(r)-\kappa_{0}\right| \in L(1, \infty) .
$$


When $a \in \mathbb{B}_{D}$, the subfunctions (superfunctions) associated with $\mathcal{L}_{a}$ are continuous (see, e.g., [16]). In the rest of paper, we will always assume that $a \in B_{D}$.

An important role will be played by the solutions of the ordinary differential equation

$$
-Q^{\prime \prime}(r)-\frac{n-1}{r} Q^{\prime}(r)+\left(\frac{\tau}{r^{2}}+a(r)\right) Q(r)=0 \quad(0<r<\infty) .
$$

When the potential $a \in \mathcal{A}_{D}$, these solutions are well known (see [17] for more references). Equation (1.10) has two specially linearly independent positive solutions $V(r)$ and $W(r)$ such that $V$ is increasing with

$$
0 \leq V(0+) \leq V(r) \quad \text { as } r \longrightarrow+\infty
$$

and $W$ is decreasing with

$$
+\infty=W(0+)>W(r) \searrow 0 \text { as } r \longrightarrow+\infty
$$

We remark that both $V(r) \varphi(\Theta)$ and $W(r) \varphi(\Theta)$ are harmonic on $C_{n}(\Omega)$ and vanish continuously on $S_{n}(\Omega)$.

Denote

$$
\iota_{\kappa}^{ \pm}=\frac{2-n \pm \sqrt{(n-2)^{2}+4(\kappa+\tau)}}{2} .
$$

When $a \in \mathbb{B}_{D}$, the normalized solutions $V(r)$ and $W(r)$ of $(1.10)$ satisfying $V(1)=W(1)=1$ have the asymptotics (see [15]):

$$
V(r) \approx r^{\iota_{\kappa}^{+}}, \quad W(r) \approx r_{\kappa}^{\iota_{\kappa}^{-}}, \quad \text { as } r \longrightarrow \infty
$$

Set

$$
x=\iota_{\mathcal{\kappa}}^{+}-\iota_{\mathcal{\kappa}}^{-}=\sqrt{(n-2)^{2}+4(\kappa+\tau)}, \quad x^{\prime}=\left.\omega(V(r), W(r))\right|_{r=1}
$$

where $\chi^{\prime}$ is their Wronskian at $r=1$.

Remark 1.1. If $a=0$ and $\Omega=\mathbf{S}_{+}^{n-1}$, then $\iota_{0}^{+}=1, \iota_{0}^{-}=1-n$ and $\varphi(\Theta)=\left(2 n s_{n}^{-1}\right)^{1 / 2} \cos \theta_{1}$, where $s_{n}=2 \pi^{n / 2}\{\Gamma(n / 2)\}^{-1}$ is the surface area of $\mathbf{S}^{n-1}$.

We recall that

$$
C_{1} V(r) W(t) \varphi(\Theta) \varphi(\Phi) \leq G_{\Omega}^{a}(P, Q) \leq C_{2} V(r) W(t) \varphi(\Theta) \varphi(\Phi)
$$

or

$$
C_{1} V(t) W(r) \varphi(\Theta) \varphi(\Phi) \leq G_{\Omega}^{a}(P, Q) \leq C_{2} V(t) W(r) \varphi(\Theta) \varphi(\Phi)
$$


for any $P=(r, \Theta) \in C_{n}(\Omega)$ and any $Q=(t, \Phi) \in C_{n}(\Omega)$ satisfying $0<r / t \leq 4 / 5$ or $0<t / r \leq$ $4 / 5$, where $C_{1}$ and $C_{2}$ are two positive constants (see Escassut et al. [11, Chapter 11], and for $a=0$, see Azarin [6, Lemma 1], Essén, and Lewis [18, Lemma 2]).

The remainder of the paper is organized as follows: in Section 2 we will give our main theorems; in Section 3, some necessary lemmas are given; in Section 4, we will prove the main results.

\section{Statement of the Main Results}

In this section, we will state our main results. Before passing to our main results, we need some definitions.

Martin introduced the so-called Martin functions associated with the Laplace operator (see Brelot [19] or Martin [20]). Inspired by his spirit, we define the Martin function $M_{\Omega}^{a}$ associated with the stationary Schrödinger operator as follows:

$$
M_{\Omega}^{a}(P, Q)=\frac{G_{\Omega}^{a}(P, Q)}{G_{\Omega}^{a}\left(P_{0}, Q\right)} \quad\left(P, Q \in C_{n}(\Omega) \times C_{n}(\Omega) \backslash\left(P_{0}, P_{0}\right)\right)
$$

which will be called the generalized Martin Kernel of $C_{n}(\Omega)$ (relative to $P_{0}$ ). If $Q=P_{0}$, the above quotient is interpreted as 0 (for $a=0$, refer to Armitage and Gardiner [3]).

It is well known that the Martin boundary $\Delta$ of $C_{n}(\Omega)$ is the set $\partial C_{n}(\Omega) \cup\{\infty\}$. When we denote the Martin kernel associated with the stationary Schrödinger operator by $M_{\Omega}^{a}(P, Q)\left(P \in C_{n}(\Omega), Q \in \partial C_{n}(\Omega) \cup\{\infty\}\right)$ with respect to a reference point chosen suitably, we see

$$
M_{\Omega}^{a}(P, \infty)=V(r) \varphi(\Theta), \quad M_{\Omega}^{a}(P, O)=K W(r) \varphi(\Theta)
$$

for any $P \in C_{n}(\Omega)$, where $O$ is the origin of $\mathbf{R}^{n}$ and $K$ a positive constant.

Let $E$ be a subset of $C_{n}(\Omega)$ and let $u$ be a nonnegative superfunction on $C_{n}(\Omega)$. The reduced function of $u$ is defined by

$$
R_{u}^{E}(P)=\inf \left\{v(P): v \in \Phi_{u}^{E}\right\}
$$

where $\Phi_{u}^{E}=\left\{v \in \operatorname{SpH}\left(a, C_{n}(\Omega)\right): v \geq 0\right.$ on $C_{n}(\Omega), v \geq u$ on $\left.E\right\}$. We define the regularized reduced function $\widehat{R}_{u}^{E}$ of $u$ relative to $E$ as follows:

$$
\widehat{R}_{u}^{E}(P)=\lim _{P^{\prime} \rightarrow P} \inf R_{u}^{E}\left(P^{\prime}\right)
$$

It is easy to see that $\widehat{R}_{u}^{E}$ is a superfunction on $C_{n}(\Omega)$.

If $E \subseteq C_{n}(\Omega)$ and $Q \in \Delta$, then the Riesz decomposition and the generalized Martin representation allow us to express $\widehat{R}_{M_{\Omega}^{a}(, Q)}^{E}$ uniquely in the form $G_{\Omega}^{a} \mu+M_{\Omega}^{a} \nu$, where $G_{\Omega}^{a} \mu$ and $M_{\Omega}^{a} v$ are the generalized Green potential and generalized Martin representation, respectively. We say that $E$ is a-minimally thin at $Q$ with respect to $C_{n}(\Omega)$ if $v(\{Q\})=0$. At last we remark 
that $\Delta_{0}=\left\{Q \in \Delta: C_{n}(\Omega)\right.$ is a-minimally thin at $\left.Q\right\}$, where $\Delta$ is the Martin boundary of $C_{n}(\Omega)$.

Now we can state our main theorems.

Theorem 2.1. Let $E \subseteq C_{n}(\Omega)$ and a fixed point $Q \in \Delta \backslash \Delta_{0}$. The following are equivalent:

(a) $E$ is a-minimally thin at $Q$;

(b) $\widehat{R}_{M_{\Omega}^{a}(\cdot, Q)}^{E} \neq M_{\Omega}^{a}(\cdot, Q)$;

(c) $\inf \left\{\widehat{R}_{M_{\Omega}^{a}(\cdot, Q)}^{E \cap \omega}: \omega\right.$ is a generalized Martin topology neighbourhood of $\left.Q\right\}=0$.

If $u$ is a positive superfunction, then we will write $\mu_{u}$ for the measure appearing in the generalized Martin representation of the greatest a-harmonic minorant of $u$.

Theorem 2.2. Let $E \subseteq C_{n}(\Omega)$ and a fixed point $Q \in \Delta \backslash \Delta_{0}$. Suppose that $Q$ is a generalized Martin topology limit of $E$. The following are equivalent:

(a) E is a-minimally thin at $Q$;

(b) there exists a positive superfunction $u$ such that

$$
\liminf _{P \rightarrow Q, P \in E} \frac{u(P)}{M_{\Omega}^{a}(P, Q)}>\mu_{u}(\{Q\})
$$

(c) there is an a-potential $u$ on $C_{n}(\Omega)$ such that

$$
\frac{u(P)}{M_{\Omega}^{a}(P, Q)} \longrightarrow \infty \quad(P \longrightarrow Q ; P \in E)
$$

A set $E$ in $\mathbf{R}^{n}$ is said to be $a$-thin at a point $Q$ if there is a fine neighborhood $U$ of $Q$ which does not intersect $E \backslash\{Q\}$. Otherwise $E$ is said to be not $a$-thin at $Q$. A set $E$ in $\mathbf{R}^{n}$ is called $a$-polar if there is a superfunction $u$ on some open set $\omega$ such that $E \subseteq\{P \in \omega: u(P)=$ $\infty\}$.

Let $E$ be a bounded subset of $C_{n}(\Omega)$. Then $\widehat{R}_{M_{\Omega}^{a}(\cdot, \infty)}(P)$ is bounded on $C_{n}(\Omega)$, and hence the greatest a-harmonic minorant of $\widehat{R}_{M_{\Omega}^{a}(, \infty)}^{E}(P)$ is zero. By the Riesz decomposition theorem there exists a unique positive measure $\lambda_{E}^{\frac{\Omega}{a}}$ associated with the stationary Schrödinger operator $\mathcal{L}_{a}$ on $C_{n}(\Omega)$ such that

$$
\widehat{R}_{M_{\Omega}^{a}(\cdot, \infty)}^{E}(P)=G_{\Omega}^{a} \lambda_{E}^{a}(P)
$$

for any $P \in C_{n}(\Omega)$, and $\lambda_{E}^{a}$ is concentrated on $B_{E}$, where

$$
B_{E}=\left\{P \in C_{n}(\Omega): E \text { is not } a \text {-thin at } P\right\} .
$$

For $a=0$, see Brelot [19] and Doob [21]. According to the Fatou's lemma, we easily know the condition (b) in Theorems 2.3 and 2.4. 
Theorem 2.3. Let $E \subseteq C_{n}(\Omega)$ and a fixed point $Q \in \Delta \backslash \Delta_{0}$. Suppose that $Q$ is a generalized Martin topology limit point of $E$. The following are equivalent:

(a) $E$ is a-minimally thin at $Q$;

(b) there is an a-potential $G_{\Omega}^{a} \mu$ such that

$$
\liminf _{P \rightarrow Q, P \in E} \frac{G_{\Omega}^{a} \mu(P)}{G_{\Omega}^{a}\left(P_{0}, P\right)}>\int M_{\Omega}^{a}(P, Q) d \mu(P)
$$

(c) there is an a-potential $G_{\Omega}^{a} \mu^{\prime}$ such that $\int M_{\Omega}^{a}(P, Q) d \mu^{\prime}(P)<\infty$ and

$$
\frac{G_{\Omega}^{a} \mu^{\prime}(P)}{G_{\Omega}^{a}\left(P_{0}, P\right)} \longrightarrow \infty \quad(P \longrightarrow Q ; P \in E) .
$$

Theorem 2.4. Let $E \subseteq C_{n}(\Omega), Q_{0} \in C_{n}(\Omega)$ and a fixed point $Q \in \Delta \backslash \Delta_{0}$. Suppose that $Q$ is a generalized Martin topology limit point of $E$. Then $E$ is a-minimally thin at $Q$ if and only if there exists a positive superfunction $u$ such that

$$
\liminf _{P \rightarrow Q, P \in E} \frac{u(P)}{G_{\Omega}^{a}\left(Q_{0}, P\right)}>\liminf _{P \rightarrow Q} \frac{u(P)}{G_{\Omega}^{a}\left(Q_{0}, P\right)}
$$

The generalized Green energy $\gamma_{\Omega}^{a}(E)$ of $\lambda_{E}^{a}$ is defined by

$$
\gamma_{\Omega}^{a}(E)=\int_{C_{n}(\Omega)}\left(G_{\Omega}^{a} \lambda_{E}^{a}\right) d \lambda_{E}^{a}
$$

Let $E$ be a subset of $C_{n}(\Omega)$ and $E_{k}=E \cap I_{k}(\Omega)$, where $I_{k}(\Omega)=\left\{P=(r, \Omega) \in C_{n}(\Omega): 2^{k} \leq\right.$ $\left.r \leq 2^{k+1}\right\}$. The previous theorems are concerned with the fixed boundary points. Next we will consider the case at infinity.

Theorem 2.5. A subset $E$ of $C_{n}(\Omega)$ is a-minimally thin at $\infty$ with respect to $C_{n}(\Omega)$ if and only if

$$
\sum_{k=0}^{\infty} r_{\Omega}^{a}\left(E_{k}\right) W\left(2^{k}\right) V\left(2^{k}\right)^{-1}<\infty
$$

A subset $E$ of $C_{n}(\Omega)$ is a-rarefied at $\infty$ with respect to $C_{n}(\Omega)$, if there exists a positive superfunction $v(P)$ in $C_{n}(\Omega)$ such that

$$
\inf _{P \in C_{n}(\Omega)} \frac{v(P)}{M_{\Omega}^{a}(P, \infty)} \equiv 0, \quad E \subset H_{v}
$$

where

$$
H_{v}=\left\{P=(r, \Theta) \in C_{n}(\Omega): v(P) \geq V(r)\right\}
$$


Theorem 2.6. A subset $E$ of $C_{n}(\Omega)$ is a-rarefied at $\infty$ with respect to $C_{n}(\Omega)$ if and only if

$$
\sum_{k=0}^{\infty} W\left(2^{k}\right) \lambda_{\Omega}^{a}\left(E_{k}\right)<\infty
$$

Remark 2.7. When $a=0$, Theorems 2.5 and 2.6 reduce to the results by Miyamoto and Yoshida [5]. When $a=0$ and $\Omega=\mathbf{S}_{+}^{n-1}$, these are exactly due to Aikawa and Essén [22].

Set

$$
c(v, a)=\inf _{P \in C_{n}(\Omega)} \frac{v(P)}{M_{\Omega}^{a}(P, \infty)}
$$

for a positive superfunction $v(P)$ on $C_{n}(\Omega)$. We immediately know that $c(v, a)<\infty$. Actually let $u(P)$ be a subfunction on $C_{n}(\Omega)$ satisfying

$$
\limsup _{P \rightarrow Q, P \in C_{n}(\Omega)} u(P) \leq 0
$$

for any $Q \in \partial C_{n}(\Omega) \backslash\{O\}$ and

$$
\sup _{P=(r, \Theta) \in C_{n}(\Omega)} \frac{u(P)}{V(r) \varphi(\Theta)}=\ell(a)<\infty
$$

Then we see $\ell(a)>-\infty$ (for $a=0$, see Yoshida [23]). If we apply this to $u=-v$, we may obtain $c(v, a)<\infty$.

Theorem 2.8. Let $v(P)$ be a positive superfunction on $C_{n}(\Omega)$. Then there exists an a-rarefied set $E$ at $\infty$ with respect to $C_{n}(\Omega)$ such that $v(P) V(r)^{-1}$ uniformly converges to $c(v, a) \varphi(\Theta)$ on $C_{n}(\Omega) \backslash E$ as $r \rightarrow \infty$, where $P=(r, \Theta) \in C_{n}(\Omega)$.

From the definition of $a$-rarefied set, for any given $a$-rarefied set $E$ at $\infty$ with respect to $C_{n}(\Omega)$ there exists a positive superfunction $v(P)$ on $C_{n}(\Omega)$ such that $v(P) V(r)^{-1} \geq 1$ on $E$ and $c(v, a)=0$. Hence $v(P) V(r)^{-1}$ does not converge to $c(v, a) \varphi(\Theta)=0$ on $E$ as $r \rightarrow \infty$.

Let $u(P)$ be a subfunction on $C_{n}(\Omega)$ satisfying (2.18) and (2.19). Then

$$
v(P)=\ell(a) V(r) \varphi(\Theta)-u(P), \quad\left(P=(r, \Theta) \in C_{n}(\Omega)\right)
$$

is a positive superfunction on $C_{n}(\Omega)$ such that $c(v, a)=0$. If we apply Theorem 2.8 to this $v(P)$, then we obtain the following corollary.

Corollary 2.9. Let $u(P)$ be a subfunction on $C_{n}(\Omega)$ satisfying (2.18) and (2.19) for $P \in C_{n}(\Omega)$. Then there exists an a-rarefied set $E$ at $\infty$ with respect to $C_{n}(\Omega)$ such that $v(P) V(r)^{-1}$ uniformly converges to $\ell(a) \varphi(\Theta)$ on $C_{n}(\Omega) \backslash E$ as $r \rightarrow \infty$, where $P=(r, \Theta) \in C_{n}(\Omega)$. $S^{n-1}$.

A cone $C_{n}\left(\Omega^{\prime}\right)$ is called a subcone of $C_{n}(\Omega)$ if $\overline{\Omega^{\prime}} \subset \Omega$, where $\overline{\Omega^{\prime}}$ is the closure of $\Omega^{\prime} \subset$ 
Theorem 2.10. Let $E$ be a subset of $C_{n}(\Omega)$. If $E$ is an a-rarefied set at $\infty$ with respect to $C_{n}(\Omega)$, then $E$ is a-minimally thin at $\infty$ with respect to $C_{n}(\Omega)$. If $E$ is contained in a subcone of $C_{n}(\Omega)$ and $E$ is a-minimally thin at $\infty$ with respect to $C_{n}(\Omega)$, then $E$ is an a-rarefied set at $\infty$ with respect to $C_{n}(\Omega)$.

\section{Some Lemmas}

In our arguments we need the following results.

Lemma 3.1. Let $E_{1}, E_{2}, \ldots, E_{m} \subseteq C_{n}(\Omega)$ and $Q \in \Delta$.

(i) If $E_{1} \subseteq E_{2}$ and $E_{2}$ is a-minimally thin at $Q$, then $E_{1}$ is a-minimally thin at $Q$.

(ii) If $E_{1}, E_{2}, \ldots, E_{m}$ are a-minimally thin at $Q$, then $\bigcup_{k=1}^{m} E_{k}$ is a-minimally thin at $Q$.

(iii) If $E_{1}$ is a-minimally thin at $Q$, then there is an open subset $E$ of $C_{n}(\Omega)$ such that $E_{1} \subseteq E$ and $E$ is a-minimally thin at $Q$.

Proof. Since $\widehat{R}_{M_{\Omega}^{a}(\cdot, Q)}^{E_{1}} \leq \widehat{R}_{M_{\Omega}^{a}(\cdot, Q)}^{E_{2}}$, we see (i) holds. To prove (ii) we note that $\widehat{R}_{M_{\Omega}^{a}(\cdot, Q)}^{E_{k}}$ is an $a$-potential for each $k$ and

$$
\sum_{k=1}^{m} \widehat{R}_{M_{\Omega}^{a}(\cdot, Q)}^{E_{k}} \geq M_{\Omega}^{a}(\cdot, Q) \quad \text { quasi everywhere on } \bigcup_{k=1}^{m} E_{k}
$$

so $\widehat{R}_{M_{\Omega}^{a}(,, Q)}^{\bigcup_{k} E_{k}}$ is an $a$-potential. Finally, to prove (iii), let $u=\widehat{R}_{M_{\Omega}^{a}(,, Q)}^{E_{1}}$. Then $u$ is an $a$-potential and $u \geq M_{\Omega}^{a}(\cdot, Q)$ on $E_{1} \backslash F$ for some $a$-polar set $F$. Let $v$ be a nonzero $a$-potential such that $v=\infty$ on $F$, and let

$$
Z=\left\{P \in C_{n}(\Omega): u(P)+v(P) \geq M_{\Omega}^{a}(P, Q)\right\}
$$

Then $Z$ is open, $E_{1} \subseteq Z$ and $R_{M_{\Omega}^{a}(\cdot, Q)}^{Z} \leq u+v$, so $R_{M_{\Omega}^{a}(\cdot, Q)}^{Z}$ is an $a$-potential and $Z$ is $a$-minimally thin at $Q$.

Lemma 3.2 (see [24]). Consider

$$
\begin{aligned}
& \frac{\partial G_{\Omega}^{a}(P, Q)}{\partial n_{Q}} \approx t^{-1} V(t) W(r) \varphi(\Theta) \frac{\partial \varphi(\Phi)}{\partial n_{\Phi}} \\
& \frac{\partial G_{\Omega}^{a}(P, Q)}{\partial n_{Q}} \approx V(r) t^{-1} W(t) \varphi(\Theta) \frac{\partial \varphi(\Phi)}{\partial n_{\Phi}}
\end{aligned}
$$

for any $P=(r, \Theta) \in C_{n}(\Omega)$ and any $Q=(t, \Phi) \in S_{n}(\Omega)$ satisfying $0<t / r \leq 4 / 5$ (resp., $0<r / t \leq$ 4/5). In addition,

$$
\frac{\partial G_{\Omega}^{0}(P, Q)}{\partial n_{Q}} \lesssim \frac{\varphi(\Theta)}{t^{n-1}} \frac{\partial \varphi(\Phi)}{\partial n_{\Phi}}+\frac{r \varphi(\Theta)}{|P-Q|^{n}} \frac{\partial \varphi(\Phi)}{\partial n_{\Phi}}
$$

for any $P=(r, \Theta) \in C_{n}(\Omega)$ and any $Q=(t, \Phi) \in S_{n}(\Omega ;((4 / 5) r,(5 / 4) r))$. 
Lemma 3.3 (see [24]). Let $\mu$ be a positive measure on $C_{n}(\Omega)$ such that there is a sequence of points $P_{i}=\left(r_{i}, \Theta_{i}\right) \in C_{n}(\Omega), r_{i} \rightarrow \infty(i \rightarrow \infty)$ satisfying

$$
G_{\Omega}^{a} \mu\left(P_{i}\right)=\int_{C_{n}(\Omega)} G_{\Omega}^{a}\left(P_{i}, Q\right) d \mu(t, \Phi)<\infty \quad\left(i=1,2,3, \ldots ; Q=(t, \Phi) \in C_{n}(\Omega)\right) .
$$

Then for a positive number $\ell$,

$$
\begin{gathered}
\int_{C_{n}(\Omega ;(\ell, \infty))} W(t) \varphi(\Phi) d \mu(t, \Phi)<\infty, \\
\lim _{R \rightarrow \infty} \frac{W(R)}{V(R)} \int_{C_{n}(\Omega ;(0, R))} V(t) \varphi(\Phi) d \mu(t, \Phi)=0 .
\end{gathered}
$$

Lemma 3.4 (see [24]). Let $v$ be a positive measure on $S_{n}(\Omega)$ such that there is a sequence of points $P_{i}=\left(r_{i}, \Theta_{i}\right) \in C_{n}(\Omega), r_{i} \rightarrow \infty(i \rightarrow \infty)$ satisfying

$$
\int_{S_{n}(\Omega)} \frac{\partial G_{\Omega}^{a}\left(P_{i}, Q\right)}{\partial n_{Q}} d v(Q)<\infty \quad\left(i=1,2,3, \ldots ; Q=(t, \Phi) \in C_{n}(\Omega)\right) .
$$

Then for a positive number $\ell$,

$$
\begin{gathered}
\int_{S_{n}(\Omega ;(\ell, \infty))} W(t) t^{-1} \frac{\partial \varphi(\Phi)}{\partial n_{\Phi}} d v(t, \Phi)<\infty \\
\lim _{R \rightarrow \infty} \frac{W(R)}{V(R)} \int_{S_{n}(\Omega ;(0, R))} V(t) t^{-1} \frac{\partial \varphi(\Phi)}{\partial n_{\Phi}} d v(t, \Phi)=0
\end{gathered}
$$

Lemma 3.5. Let $\mu$ be a positive measure on $C_{n}(\Omega)$ for which $G_{\Omega}^{a} \mu(P)$ is defined. Then for any positive number $A$ the set

$$
\left\{P=(r, \Theta) \in C_{n}(\Omega): G_{\Omega}^{a} \mu(P) \geq A V(r) \varphi(\Theta)\right\}
$$

is a-minimally thin at $\infty$ with respect to $C_{n}(\Omega)$.

Lemma 3.6. Let $v(P)$ be a positive superfunction on $C_{n}(\Omega)$ and put

$$
c(v, a)=\inf _{P \in C_{n}(\Omega)} \frac{v(P)}{M_{\Omega}^{a}(P, \infty)}, \quad c_{O}(v, a)=\inf _{P \in C_{n}(\Omega)} \frac{v(P)}{M_{\Omega}^{a}(P, O)} .
$$

Then there are a unique positive measure $\mu$ on $C_{n}(\Omega)$ and a unique positive measure $v$ on $S_{n}(\Omega)$ such that

$$
\begin{aligned}
v(P)= & c(v, a) M_{\Omega}^{a}(P, \infty)+c_{O}(v, a) M_{\Omega}^{a}(P, O) \\
& +\int_{C_{n}(\Omega)} G_{\Omega}^{a}(P, Q) d \mu(Q)+\int_{S_{n}(\Omega)} \frac{\partial G_{\Omega}^{a}(P, Q)}{\partial n_{Q}} d v(Q),
\end{aligned}
$$

where $\partial / \partial n_{Q}$ denotes the differentiation at $Q$ along the inward normal into $C_{n}(\Omega)$. 
Proof. By the Riesz decomposition theorem, we have a unique measure $\mu$ on $C_{n}(\Omega)$ such that

$$
v(P)=\int_{C_{n}(\Omega)} G_{\Omega}^{a}(P, Q) d \mu(Q)+h(P) \quad\left(P \in C_{n}(\Omega)\right),
$$

where $h$ is the greatest a-harmonic minorant of $v$ on $C_{n}(\Omega)$. Furthermore, by the generalized Martin representation theorem (Lemma 3.8) we have another positive measure $v^{\prime}$ on $\partial C_{n}(\Omega) \cup$ $\{\infty\}$ satisfying

$$
\begin{aligned}
h(P)= & \int_{\partial C_{n}(\Omega) \cup\{\infty\}} M_{\Omega}^{a}(P, Q) d v^{\prime}(Q) \\
= & M_{\Omega}^{a}(P, \infty) v^{\prime}(\{\infty\})+M_{\Omega}^{a}(P, O) v^{\prime}(\{O\}) \\
& +\int_{S_{n}(\Omega)} M_{\Omega}^{a}(P, Q) d v^{\prime}(Q) \quad\left(P \in C_{n}(\Omega)\right) .
\end{aligned}
$$

We know from (3.11) that $v^{\prime}(\{\infty\})=c(v, a)$ and $v^{\prime}(\{O\})=c_{O}(v, a)$.

Since

$$
M_{\Omega}^{a}(P, Q)=\lim _{P_{1} \rightarrow Q, P_{1} \in C_{n}(\Omega)} \frac{G_{\Omega}^{a}\left(P, P_{1}\right)}{G_{\Omega}^{a}\left(P_{0}, P_{1}\right)}=\frac{\left(\partial G_{\Omega}^{a}(P, Q)\right) / \partial n_{Q}}{\left(\partial G_{\Omega}^{a}\left(P_{0}, Q\right)\right) / \partial n_{Q}}
$$

where $P_{0}$ is a fixed reference point of the generalized Martin kernel, we also obtain

$$
h(P)=c(v, a) M_{\Omega}^{a}(P, \infty)+c_{O}(v, a) M_{\Omega}^{a}(P, O)+\int_{S_{n}(\Omega)} \frac{\partial G_{\Omega}^{a}(P, Q)}{\partial n_{Q}} d v(Q) \quad\left(P \in C_{n}(\Omega)\right)
$$

by taking

$$
d v(Q)=\left\{\frac{\partial G_{\Omega}^{a}\left(P_{0}, Q\right)}{\partial n_{Q}}\right\}^{-1} d v^{\prime}(Q) \quad\left(Q \in S_{n}(\Omega)\right)
$$

Hence by (3.13) and (3.16) we get the required.

Lemma 3.7. Let $E$ be a bounded subset of $C_{n}(\Omega)$, and let $u(P)$ be a positive superfunction on $C_{n}(\Omega)$ such that $u(P)$ is represented as

$$
u(P)=\int_{C_{n}(\Omega)} G_{\Omega}^{a}(P, Q) d \mu_{u}(Q)+\int_{S_{n}(\Omega)} \frac{\partial G_{\Omega}^{a}(P, Q)}{\partial n_{Q}} d v_{u}(Q)
$$

with two positive measures $\mu_{u}(Q)$ and $v_{u}(Q)$ on $C_{n}(\Omega)$ and $S_{n}(\Omega)$, respectively, and satisfies $u(P) \geq 1$ for any $P \in E$. Then 


$$
\lambda_{\Omega}^{a}(E) \leq \int_{C_{n}(\Omega)} V(t) \varphi(\Phi) d \mu_{u}(t, \Phi)+\int_{S_{n}(\Omega)} V(t) t^{-1} \varphi(\Phi) d v_{u}(t, \Phi) .
$$

When $u(P)=\widehat{R}_{1}^{E}(P)\left(P \in C_{n}(\Omega)\right)$, the equality holds in (3.19).

Proof. Since $\lambda_{E}^{a}$ is concentrated on $B_{E}$ and $u(P) \geq 1$ for any $P \in B_{E}$, we see that

$$
\begin{aligned}
\lambda_{\Omega}^{a}(E) & =\int_{C_{n}(\Omega)} d \lambda_{E}^{a}(P) \leq \int_{C_{n}(\Omega)} u(P) d \lambda_{E}^{a}(P) \\
& =\int_{C_{n}(\Omega)} \widehat{R}_{M_{\Omega}^{a}(\cdot, \infty)}^{E} d \mu_{u}(Q)+\int_{S_{n}(\Omega)}\left(\int_{C_{n}(\Omega)} \frac{\partial G_{\Omega}^{a}(P, Q)}{\partial n_{Q}} d \lambda_{E}^{a}(P)\right) d v_{u}(Q) .
\end{aligned}
$$

In addition, we have

$$
\widehat{R}_{M_{\Omega}^{a}(\cdot, \infty)}^{E}(Q) \leq M_{\Omega}^{a}(Q, \infty)=V(t) \varphi(\Phi) \quad\left(Q=(t, \Phi) \in C_{n}(\Omega)\right)
$$

Since

$$
\int_{C_{n}(\Omega)} \frac{\partial G_{\Omega}^{a}(P, Q)}{\partial n_{Q}} d \lambda_{E}^{a}(P) \leq \liminf _{\rho \rightarrow 0} \frac{1}{\rho} \int_{C_{n}(\Omega)} G_{\Omega}^{a}\left(P, P_{\rho}\right) d \lambda_{E}^{a}(P)
$$

for any $Q \in S_{n}(\Omega)$, where $P_{\rho}=\left(r_{\rho}, \Theta_{\rho}\right)=Q+\rho n_{Q} \in C_{n}(\Omega)$ and $n_{Q}$ is the inward normal unit vector at $Q$, and

$$
\int_{C_{n}(\Omega)} G_{\Omega}^{a}\left(P, P_{\rho}\right) d \lambda_{E}^{a}(P)=\widehat{R}_{M_{\Omega}^{a}(\cdot, \infty)}^{E}\left(P_{\rho}\right) \leq M_{\Omega}^{a}\left(P_{\rho}, \infty\right)=V\left(r_{\rho}\right) \varphi\left(\Theta_{\rho}\right),
$$

we have

$$
\int_{C_{n}(\Omega)} \frac{\partial G_{\Omega}^{a}(P, Q)}{\partial n_{Q}} d \lambda_{E}^{a}(P) \leq V(t) t^{-1} \frac{\partial \varphi(\Phi)}{\partial n_{\Phi}}
$$

for any $Q=(t, \Phi) \in S_{n}(\Omega)$. Thus (3.19) follows from (3.20), (3.21), and (3.24). Because $\widehat{R}_{1}^{E}(P)$ is bounded on $C_{n}(\Omega), u(P)$ has the expression (3.18) by Lemma 3.6 when $u(P)=\widehat{R}_{1}^{E}(P)$. Then the equalities in (3.20) hold because $\widehat{R}_{1}^{E}(P)=1$ for any $P \in B_{E}$ (Doob [21, page 169]). Hence we claim if

$$
\begin{gathered}
\mu_{u}\left(\left\{P \in C_{n}(\Omega): \widehat{R}_{M_{\Omega}^{a}(\cdot, \infty)}^{E}(P)<M_{\Omega}^{a}(P, \infty)\right\}\right)=0, \\
v_{u}\left(\left\{Q=(t, \Phi) \in S_{n}(\Omega): \int_{C_{n}(\Omega)} \frac{\partial G_{\Omega}^{a}(P, Q)}{\partial n_{Q}} d \lambda_{E}^{a}(P)<V(t) t^{-1} \frac{\partial \varphi(\Phi)}{\partial n_{\Phi}}\right\}\right)=0,
\end{gathered}
$$

then the equality in (3.19) holds. 
Abstract and Applied Analysis

To see (3.25) we remark that

$$
\begin{gathered}
\left\{P \in C_{n}(\Omega): \widehat{R}_{M_{\Omega}^{a}(, \infty)}^{E}(P)<M_{\Omega}^{a}(P, \infty)\right\} \subset\left(C_{n}(\Omega)\right) \backslash B_{E}, \\
\mu_{u}\left(C_{n}(\Omega) \backslash B_{E}\right)=0 .
\end{gathered}
$$

To prove (3.26) we set

$$
\begin{gathered}
B_{E}^{\prime}=\left\{Q \in S_{n}(\Omega): E \text { is not } a \text {-minimally thin at } Q\right\}, \\
e=\left\{P \in E: \widehat{R}_{M_{\Omega}^{a}(\cdot, \infty)}^{E}(P)<M_{\Omega}^{a}(P, \infty)\right\} .
\end{gathered}
$$

Then $e$ is an $a$-polar set, and hence

$$
\widehat{R}_{M_{\Omega}^{a}(\cdot, Q)}^{E}=\widehat{R}_{M_{\Omega}^{a}(\cdot, Q)}^{E \backslash e}
$$

for any $Q \in S_{n}(\Omega)$. Consequently, for any $Q \in B_{E^{\prime}}^{\prime} E \backslash e$ is not also $a$-minimally thin at $Q$, and so

$$
\int_{C_{n}(\Omega)} M_{\Omega}^{a}(P, Q) d \eta(P)=\liminf _{P^{\prime} \rightarrow Q, P^{\prime} \in E \backslash e} \int_{C_{n}(\Omega)} M_{\Omega}^{a}\left(P, P^{\prime}\right) d \eta(P)
$$

for any positive measure $\eta$ on $C_{n}(\Omega)$, where

$$
M_{\Omega}^{a}\left(P, P^{\prime}\right)=\frac{G_{\Omega}^{a}\left(P, P^{\prime}\right)}{G_{\Omega}^{a}\left(P_{0}, P^{\prime}\right)} \quad\left(P, P^{\prime} \in C_{n}(\Omega)\right) .
$$

Take $\eta=\lambda_{E}^{a}$ in (3.30). Since

$$
\lim _{P \rightarrow Q, P \in C_{n}(\Omega)} \frac{M_{\Omega}^{a}(P, \infty)}{G_{\Omega}^{a}\left(P_{0}, P\right)}=V(t) t^{-1} \frac{\partial \varphi(\Phi)}{\partial n_{\Phi}}\left\{\frac{\partial G_{\Omega}^{a}\left(P_{0}, Q\right)}{\partial n_{Q}}\right\}^{-1}, \quad\left(Q=(t, \Phi) \in S_{n}(\Omega)\right)
$$

we obtain from (3.15)

$$
\int_{C_{n}(\Omega)} \frac{\partial G_{\Omega}^{a}(P, Q)}{\partial n_{\Phi}} d \lambda_{E}^{a}(P)=V(t) t^{-1} \frac{\partial \varphi(\Phi)}{\partial n_{\Phi}} \liminf _{P^{\prime} \rightarrow Q, P^{\prime} \in E \backslash e} \int_{C_{n}(\Omega)} \frac{G_{\Omega}^{a}\left(P, P^{\prime}\right)}{M_{\Omega}^{a}\left(P^{\prime}, \infty\right)} d \lambda_{E}^{a}(P)
$$

for any $Q \in(t, \Phi) \in B_{E}^{\prime}$. Since

$$
\int_{C_{n}(\Omega)} \frac{G_{\Omega}^{a}\left(P, P^{\prime}\right)}{M_{\Omega}^{a}\left(P^{\prime}, \infty\right)} d \lambda_{E}^{a}(P)=\frac{1}{M_{\Omega}^{a}\left(P^{\prime}, \infty\right)} \widehat{R}_{M_{\Omega}^{a}(\cdot, \infty)}^{E}\left(P^{\prime}\right)=1
$$


for any $P^{\prime} \in E \backslash e$, we have

$$
\int_{C_{n}(\Omega)} \frac{\partial G_{\Omega}^{a}(P, Q)}{\partial n_{Q}} d \lambda_{E}^{a}(P)=V(t) t^{-1} \frac{\partial \varphi(\Phi)}{\partial n_{\Phi}}
$$

for any $Q=(t, \Phi) \in B_{E}^{\prime}$, which shows

$$
\left\{Q=(t, \Phi) \in S_{n}(\Omega): \int_{C_{n}(\Omega)} \frac{\partial G_{\Omega}^{a}(P, Q)}{\partial n_{Q}} d \lambda_{E}^{a}(P)<V(t) t^{-1} \frac{\partial \varphi(\Phi)}{\partial n_{\Phi}}\right\} \subset S_{n}(\Omega) \backslash B_{E}^{\prime}
$$

Let $h$ be the greatest a-harmonic minorant of $u(P)=\widehat{R}_{1}^{E}(P)$, and let $v_{u}^{\prime}$ be the generalized Martin representing measure of $h$. We claim if

$$
\widehat{R}_{h}^{E}(P)=h
$$

on $C_{n}(\Omega)$, then $v_{u}^{\prime}\left(S_{n}(\Omega) \backslash B_{E}^{\prime}\right)=0$. Since

$$
d v_{u}^{\prime}(Q)=\frac{\partial G_{\Omega}^{a}\left(P_{0}, Q\right)}{\partial n_{Q}} d v_{u}(Q) \quad\left(Q \in S_{n}(\Omega)\right)
$$

from (3.15), we also have $v_{u}\left(S_{n}(\Omega) \backslash B_{E}^{\prime}\right)=0$, which gives (3.26) from (3.36).

To prove (3.37), we set $u^{*}=\widehat{R}_{1}^{E}(P)-h$. Then

$$
u^{*}+h=\widehat{R}_{1}^{E}=\widehat{R}_{u^{*}+h}^{E} \leq \widehat{R}_{u^{*}}^{E}+\widehat{R}_{h^{\prime}}^{E}
$$

and hence

$$
\widehat{R}_{h}^{E}-h \geq u^{*}-\widehat{R}_{u^{*}}^{E} \geq 0,
$$

from which (3.37) follows.

Lemma 3.8 (the generalized Martin representation). If $u$ is a positive a-harmonic function on $C_{n}(\Omega)$, then there exists a measure $\mu_{u}$ on $\Delta$, uniquely determined by $u$, such that $\mu_{u}\left(\Delta_{0}\right)=0$ and

$$
u(P)=\int_{\Delta} M_{\Omega}^{a}(P, Q) d \mu_{u}(Q) \quad\left(P \in C_{n}(\Omega)\right)
$$

where $\Delta_{0}$ is the same as the previous statement.

Remark 3.9. Following the same method of Armitage and Gardiner [3] for Martin representation we may easily prove Lemma 3.8. 


\section{Proofs of the Main Theorems}

Proof of Theorem 2.1. First we assume that (b) holds, and let $u=\widehat{R}_{M_{\Omega}^{a}(\cdot, Q)}^{E}$. Since $M_{\Omega}^{a}(\cdot, Q)$ is minimal, the Riesz decomposition of $u$ is of the form $v+\ell M_{\Omega}^{a}(\cdot, Q)$, where $v$ is an a-potential associated with the stationary Schrödinger operator on $C_{n}(\Omega)$ and $0<\ell<1$. Since $u=$ $M_{\Omega}^{a}(\cdot, Q)$ quasieverywhere on $E$ and $\widehat{R}_{v}^{E}+\ell u=v+\ell M_{\Omega}^{a}(\cdot, Q)=u$ quasieverywhere on $E$,

$$
\widehat{R}_{M_{\Omega}^{a}(\cdot, Q)}^{E}=\widehat{R}_{u}^{E} \leq \widehat{R}_{v}^{E}+\ell u \leq v+\ell M_{\Omega}^{a}(\cdot, Q)=\widehat{R}_{M_{\Omega}^{a}(\cdot, Q)}^{E} .
$$

Hence $\ell\left(M_{\Omega}^{a}(\cdot, Q)-u\right) \equiv 0$, so $\ell=0$ by the hypothesis and (a) holds.

Next we assume (a) holds, and let $\omega_{m}$ be a decreasing sequence of compact neighborhoods of $Q$ in the Martin topology such that $\bigcap_{m} \omega_{m}=\{Q\}$. Then $\widehat{R}_{M_{\Omega}^{a}(\cdot, Q)}^{E \bigcap \omega_{m}}$ is a-harmonic on $C_{n}(\Omega) \backslash \omega_{m}$, and the decreasing sequence $\left\{\widehat{R}_{M_{\Omega}^{a}(\cdot, Q)}^{E \bigcap \omega_{m}}\right\}$ has a limit $h$ which is a-harmonic on $C_{n}(\Omega)$. Since $h$ is majorized by $\widehat{R}_{M_{\Omega}^{a}(\cdot, Q)}^{E}$, it follows that $h \equiv 0$ and (c) holds.

Finally we assume (c) holds, then there is a Martin topology neighborhood $\omega$ of $Q$ such that $\widehat{R}_{M_{\Omega}^{a}(\cdot, Q)}^{E \bigcap \omega} \neq M_{\Omega}^{a}(\cdot, Q)$. Since (b) implies (a), the set $E \bigcap \omega$ is $a$-minimally thin at $Q$ and so $\widehat{R}_{M_{\Omega}^{a}(\cdot, Q)}^{E \cap \omega}$ is an $a$-potential. Then $\widehat{R}_{M_{\Omega}^{a}(\cdot, Q)}^{E}$ is an $a$-potential and we yield (b).

Proof of Theorem 2.2. Obviously we see that (c) implies (b). If (b) holds, then there exist $\ell>$ $\mu_{u}(\{Q\})$ and a Martin topology neighborhood $\omega$ of $Q$ such that $u \geq \ell M_{\Omega}^{a}(\cdot, Q)$ on $E \bigcap \omega$. If $\widehat{R}_{M_{\Omega}^{a}(\cdot, Q)}^{E \cap \omega}=M_{\Omega}^{a}(\cdot, Q)$, then $u \geq \widehat{R}_{u}^{E \bigcap \omega} \geq \ell M_{\Omega}^{a}(\cdot, Q)$, and this yields contradictory conclusion that $\mu_{u}=\ell \delta_{Q}+\mu_{u-\ell M_{\Omega}^{a}(\cdot, Q)}>\mu_{u}(\{Q\}) \delta_{Q}$, where $\delta_{Q}$ is the unit measure with support $\{Q\}$. Hence $\widehat{R}_{M_{\Omega}^{a}(\cdot, Q)}^{E \bigcap \omega} \neq M_{\Omega}^{a}(\cdot, Q)$. Thus $E \bigcap \omega$ is a-minimally thin at $Q$, and so (a) holds.

Finally we assume (a) holds. By Lemma 3.1 there is an open subset $U$ of $C_{n}(\Omega)$ such that $E \subseteq U$ and $U$ is a-minimally thin at $Q$. By Theorem 2.1 there is a sequence $\left\{\omega_{m}\right\}$ of Martin topology open neighborhoods of $Q$ such that $\widehat{R}_{M_{\Omega}^{a}(\cdot, Q)}^{E \cap \omega_{m}}\left(P_{0}\right)<2^{-m}$. The function $u_{1}=$ $\sum_{n} \hat{R}_{M_{\Omega}^{a}(\cdot, Q)^{\prime}}^{U \cap \omega_{m}}$ being a sum of a-potentials, is an a-potential since $u_{1}\left(P_{0}\right)<\infty$. Further, since $\widehat{R}_{M_{\Omega}^{a}(\cdot, Q)}^{E} \bigcap_{m}=M_{\Omega}^{a}(\cdot, Q)$ on the open set $E \bigcap \omega_{m}$,

$$
\frac{u_{1}(P)}{M_{\Omega}^{a}(P, Q)} \longrightarrow \infty \quad(P \longrightarrow Q ; P \in U)
$$

and so (c) holds.

Proof of Theorem 2.3. Clearly (c) implies (b). To prove that (b) implies (a), we suppose that (b) holds and choose $A$ such that

$$
\liminf _{P \rightarrow Q, P \in E} \frac{G_{\Omega}^{a} \mu(P)}{G_{\Omega}^{a}\left(P_{0}, P\right)}>A>\int M_{\Omega}^{a}(\cdot, Q) d \mu
$$


Then $G_{\Omega}^{a} \mu>A G_{\Omega}^{a}\left(P_{0}, \cdot\right)$ on $E \cap \omega$ for some Martin topology neighborhood $\omega$ of $Q$. If $v$ denotes the swept measure of $\delta_{P_{0}}$ onto $E \cap \omega$, where $\delta_{P_{0}}$ is the unit measure with support $\left\{P_{0}\right\}$, then it follows that

$$
G_{\Omega}^{a} \mu \geq A \widehat{R}_{G_{\Omega}^{a}\left(P_{0}, \cdot\right)}^{E \cap \omega}=A G_{\Omega}^{a} \mathcal{v}
$$

on $C_{n}(\Omega)$. Let $\left\{K_{n}\right\}$ be a sequence of compact subsets of $C_{n}(\Omega)$ such that $\bigcup_{n} K_{n}=C_{n}(\Omega)$, and let $G_{\Omega}^{a} \mu_{n}$ denote the a-potential $\widehat{R}_{M_{\Omega}^{a}(\cdot, Q)}^{K_{n}}$. Then

$$
\int \widehat{R}_{M_{\Omega}^{a}(\cdot, Q)}^{K_{n}} d v=\int G_{\Omega}^{a} v d \mu_{n} \leq A^{-1} \int G_{\Omega}^{a} \mu d \mu_{n}=A^{-1} \int \widehat{R}_{M_{\Omega}^{a}(\cdot, Q)}^{K_{n}} d \mu
$$

Letting $n \rightarrow \infty$, we see from our choice of $A$ that

$$
\widehat{R}_{M_{\Omega}^{a}(\cdot, Q)}^{E \cap \omega}\left(P_{0}\right)=\int M_{\Omega}^{a}(\cdot, Q) d v \leq A^{-1} \int M_{\Omega}^{a}(\cdot, Q) d \mu<1=M_{\Omega}^{a}\left(P_{0}, Q\right),
$$

then $E \cap \omega$ is a-minimally thin at $Q$ by Theorem 2.1, and so (a) holds.

Next we suppose that (a) holds. By Lemma 3.1 there is an open subset $U$ of $C_{n}(\Omega)$ such that $E \subseteq U$ and $U$ is a-minimally thin at $Q$. By Theorem 2.1 there is a sequence $\left\{\omega_{n}\right\}$ of Martin topology open neighborhoods of $Q$ such that

$$
\sum_{n} \widehat{R}_{M_{\Omega}^{a}(\cdot, Q)}^{U \cap \omega_{n}}\left(P_{0}\right)<\infty
$$

Let $\mu^{\prime}=\sum_{n} v_{n}$, where $v_{n}$ is swept measure of $\delta_{P_{0}}$ onto $U \cap \omega_{n}$. Then

$$
\int M_{\Omega}^{a}(P, Q) d \mu^{\prime}(P)=\sum_{n} \int M_{\Omega}^{a}(P, Q) d v_{n}(P)=\sum_{n} \widehat{R}_{M_{\Omega}^{a}(\cdot, Q)}^{U \cap \omega_{n}}\left(P_{0}\right)<\infty,
$$

and (2.10) holds since

$$
G_{\Omega}^{a} v_{n}=\widehat{R}_{G_{\Omega}^{a}\left(P_{0,}\right)}^{U \cap \omega_{n}}=G_{\Omega}^{a}\left(P_{0}, \cdot\right)
$$

on the open set $U \cap \omega_{n}$, so (c) holds.

Proof of Theorem 2.4. Since (2.11) is independent of the choice of $Q_{0}$, we may multiply across by $M_{\Omega}^{a}\left(Q_{0}, Q\right)$. Thus we may assume that $Q_{0}=P_{0}$ and claim that

$$
\liminf _{P \rightarrow Q} \frac{G_{\Omega}^{a} \mu(P)}{G_{\Omega}^{a}\left(P_{0}, P\right)}=\int M_{\Omega}^{a}(P, Q) d \mu(P)
$$

for any a-potential $G_{\Omega}^{a} \mu$. According to Fatou's lemma, we may yield

$$
\liminf _{P \rightarrow Q} \frac{G_{\Omega}^{a} \mu(P)}{G_{\Omega}^{a}\left(P_{0}, P\right)} \geq \int M_{\Omega}^{a}(P, Q) d \mu(P) .
$$


Since $C_{n}(\Omega)$ is not $a$-minimally thin at $Q$, we know that

$$
\liminf _{P \rightarrow Q} \frac{G_{\Omega}^{a} \mu(P)}{G_{\Omega}^{a}\left(P_{0}, P\right)}<\int M_{\Omega}^{a}(P, Q) d \mu(P)
$$

from Theorem 2.3. Hence the claim holds.

When $E$ is $a$-minimally thin at $Q$, we see from (4.10) and the condition (b) of Theorem 2.3 that (2.11) holds for some $a$-potential $u$. Conversely, if (2.11) holds, then we can choose $A$ such that

$$
\liminf _{P \rightarrow Q, P \in E} \frac{u(P)}{G_{\Omega}^{a}\left(P_{0}, P\right)}>A>\liminf _{P \rightarrow Q} \frac{u(P)}{G_{\Omega}^{a}\left(P_{0}, P\right)}
$$

and define $G_{\Omega}^{a} \mu$ by $\min \left\{u, A G_{\Omega}^{a}\left(P_{0}, \cdot\right)\right\}$. Then by (4.10)

$$
\liminf _{P \rightarrow Q, P \in E} \frac{G_{\Omega}^{a} \mu(P)}{G_{\Omega}^{a}\left(P_{0}, P\right)}=A>\liminf _{P \rightarrow Q} \frac{G_{\Omega}^{a} \mu(P)}{G_{\Omega}^{a}\left(P_{0}, P\right)}=\int M_{\Omega}^{a}(P, Q) d \mu(P),
$$

and it follows from Theorem 2.3 that $E$ is a-minimally thin at $Q$.

Proof of Theorem 2.5. By applying the Riesz decomposition theorem to the superfunction $\widehat{R}_{M_{\Omega}^{a}(\cdot, \infty)}^{E}$ on $C_{n}(\Omega)$, we have a positive measure $\mu$ on $C_{n}(\Omega)$ satisfying

$$
G_{\Omega}^{a} \mu(P)<\infty
$$

for any $P \in C_{n}(\Omega)$ and a nonnegative greatest a-harmonic minorant $H$ of $\widehat{R}_{M_{\Omega}^{a}(\cdot, \infty)}^{E}$ such that

$$
\widehat{R}_{M_{\Omega}^{a}(, \infty)}^{E}=G_{\Omega}^{a} \mu(P)+H .
$$

We remark that $M_{\Omega}^{a}(\cdot, \infty)\left(P \in C_{n}(\Omega)\right)$ is a minimal function at $\infty$. If $E$ is $a$-minimally thin at $\infty$ with respect to $C_{n}(\Omega)$, then $\widehat{R}_{M_{\Omega}^{a}(\cdot, \infty)}^{E}$ is an $a$-potential, and hence $H \equiv 0$ on $C_{n}(\Omega)$. Since

$$
\widehat{R}_{M_{\Omega}^{a}(\cdot, \infty)}^{E}(P)=M_{\Omega}^{a}(P, \infty)
$$

for any $P \in B_{E}$, we see from (4.16) that

$$
G_{\Omega}^{a} \mu(P)=M_{\Omega}^{a}(P, \infty)
$$

for any $P \in B_{E}$. Take a sufficiently large $R$ from Lemma 3.3 such that

$$
C_{2} \frac{W(R)}{V(R)} \int_{C_{n}(\Omega ;(0, R])} V(t) \varphi(\Phi) d \mu(t, \Phi)<\frac{1}{4}
$$


Then from (1.16) or (1.17),

$$
\int_{C_{n}(\Omega ;(0, R])} G_{\Omega}^{a}(P, Q) d \mu(Q)<\frac{1}{4} M_{\Omega}^{a}(P, \infty)
$$

for any $P=(r, \Theta) \in C_{n}(\Omega)$ and $r \geq(5 / 4) r$, and hence from (4.18)

$$
\int_{C_{n}(\Omega ;[R, \infty))} G_{\Omega}^{a}(P, Q) d \mu(Q) \geq \frac{3}{4} M_{\Omega}^{a}(P, \infty)
$$

for any $P=(r, \Theta) \in B_{E}$ and $r \geq(5 / 4) r$. Divide $G_{\Omega}^{a} \mu$ into three parts as follows:

$$
G_{\Omega}^{a} \mu(P)=A_{1}^{(k)}(P)+A_{2}^{(k)}(P)+A_{3}^{(k)}(P) \quad\left(P=(r, \Theta) \in C_{n}(\Omega)\right),
$$

where

$$
\begin{aligned}
& A_{1}^{(k)}(P)=\int_{C_{n}\left(\Omega ;\left(2^{k-1}, 2^{k+2}\right)\right)} G_{\Omega}^{a}(P, Q) d \mu(Q), \\
& A_{2}^{(k)}(P)=\int_{C_{n}\left(\Omega ;\left(0,2^{k-1}\right]\right)} G_{\Omega}^{a}(P, Q) d \mu(Q), \\
& A_{3}^{(k)}(P)=\int_{\left.C_{n}\left(\Omega ; 2^{k+2}, \infty\right)\right)} G_{\Omega}^{a}(P, Q) d \mu(Q) .
\end{aligned}
$$

Now we claim that there exists an integer $N$ such that

$$
B_{E} \cap \overline{I_{k}(\Omega)} \subset\left\{P=(r, \Theta) \in C_{n}(\Omega): A_{1}^{(k)}(P) \geq \frac{1}{4} V(r) \varphi(\Theta)\right\} \quad(k \geq N) .
$$

When we choose a sufficiently large integer $N_{1}$ by Lemma 3.3 such that

$$
\begin{gathered}
\frac{W\left(2^{k}\right)}{V\left(2^{k}\right)} \int_{C_{n}\left(\Omega ;\left(0,2^{k}\right]\right)} V(t) \varphi(\Phi) d \mu(t, \Phi)<\frac{1}{4 C_{2}} \quad\left(k \geq N_{1}\right) \\
\int_{C_{n}\left(\Omega ;\left[2^{k+2}, \infty\right)\right)} W(t) \varphi(\Phi) d \mu(t, \Phi)<\frac{1}{4 C_{2}} \quad\left(k \geq N_{1}\right)
\end{gathered}
$$

for any $P=(r, \Theta) \in \overline{I_{k}(\Omega)} \cap C_{n}(\Omega)$, we have from (1.16) or (1.17) that

$$
\begin{array}{ll}
A_{2}^{(k)}(P) \leq \frac{1}{4} V(r) \varphi(\Theta) & \left(k \geq N_{1}\right) \\
A_{3}^{(k)}(P) & \leq \frac{1}{4} V(r) \varphi(\Theta) \quad\left(k \geq N_{1}\right) .
\end{array}
$$


Put

$$
N=\max \left\{N_{1},\left[\frac{\log R}{\log 2}\right]+2\right\}
$$

For any $P=(r, \Theta) \in B_{E} \cap \overline{I_{k}(\Omega)}(k \geq N)$, we have from (4.21), (4.22), and (4.26) that

$$
A_{1}^{(k)}(P) \geq \int_{C_{n}(\Omega ;[R, \infty))} G_{\Omega}^{a}(P, Q) d \mu(Q)-A_{2}^{(k)}(P)-A_{3}^{(k)}(P) \geq \frac{1}{4} V(r) \varphi(\Theta),
$$

which shows (4.24).

Since the measure $\lambda_{E_{k}}^{a}$ is concentrated on $B_{E_{k}}$ and $B_{E_{k}} \subset B_{E} \cap \overline{I_{k}(\Omega)}$, finally we obtain by (4.24) that

$$
\begin{aligned}
r_{\Omega}^{a}\left(E_{k}\right) & =\int_{C_{n}(\Omega)}\left(G_{\Omega^{a}}^{a} \lambda_{E_{k}}^{a}\right) d \lambda_{E_{k}}^{a}(P) \\
& \leq \int_{B_{E_{k}}} V(r) \varphi(\Theta) d \lambda_{E_{k}}^{a}(r, \Theta) \leq 4 \int_{B_{E_{k}}} A_{1}^{(k)}(P) d \lambda_{E_{k}}^{a}(P) \\
& \leq 4 \int_{C_{n}\left(\Omega ;\left(2^{k-1}, 2^{k+2}\right)\right)}\left\{\int_{C_{n}(\Omega)} G_{\Omega}^{a}(P, Q) d \lambda_{E_{k}}^{a}(P)\right\} d \mu(Q) \\
& \leq 4 \int_{C_{n}\left(\Omega ;\left(2^{k-1}, 2^{k+2}\right)\right)} V(t) \varphi(\Phi) d \mu(t, \Phi) \quad(k \geq N),
\end{aligned}
$$

and hence

$$
\begin{aligned}
\sum_{k=N}^{\infty} r_{\Omega}^{a}\left(E_{k}\right) W\left(2^{k}\right) V\left(2^{k}\right)^{-1} & \lesssim \sum_{k=N}^{\infty} \int_{C_{n}\left(\Omega ;\left(2^{k-1}, 2^{k+2}\right)\right)} W(t) \varphi(\Phi) d \mu(t, \Phi) \\
& =\int_{C_{n}\left(\Omega ;\left(2^{N-1}, \infty\right)\right)} W(t) \varphi(\Phi) d \mu(t, \Phi)<\infty
\end{aligned}
$$

from Lemma 3.3, (1.11) and Lemma C.1 in ([11] or [13]), which gives (2.13).

Next we will prove the sufficiency. Since

$$
\widehat{R}_{M_{\Omega}^{a}(\cdot, \infty)}^{E_{k}}(Q)=M_{\Omega}^{a}(Q, \infty)
$$

for any $Q \in B_{E_{k}}$ as in (4.17), we have

$$
\gamma_{\Omega}^{a}\left(E_{k}\right)=\int_{B_{E_{k}}} M_{\Omega}^{a}(Q, \infty) d \lambda_{E_{k}}^{a}(Q) \geq V\left(2^{k}\right) \int_{B_{E_{k}}} \varphi(\Phi) d \lambda_{E_{k}}^{a}(t, \Phi) \quad\left(Q=(t, \Phi) \in C_{n}(\Omega)\right),
$$


and hence from (1.16) or (1.17), (1.11), and (1.12)

$$
\widehat{R}_{M_{\Omega}^{a}(\cdot, \infty)}^{E_{k}}(P) \leq C_{2} V(r) \varphi(\Theta) \int_{B_{E_{k}}} W(t) \varphi(\Phi) d \lambda_{E_{k}}^{a}(t, \Phi) \leq C_{2} V(r) \varphi(\Theta) V^{-1}\left(2^{k}\right) W\left(2^{k}\right) r_{\Omega}^{a}\left(E_{k}\right)
$$

for any $P=(r, \Theta) \in C_{n}(\Omega)$ and any integer $k$ satisfying $2^{k} \geq(5 / 4) r$. Define a measure $\mu$ on $C_{n}(\Omega)$ by

$$
d \mu(Q)= \begin{cases}\sum_{k=0}^{\infty} d \lambda_{E_{k}}^{a}(Q) & \left(Q \in C_{n}(\Omega ;[1, \infty))\right) \\ 0 & \left(Q \in C_{n}(\Omega ;(0,1))\right)\end{cases}
$$

Then from (2.13) and (4.33)

$$
G_{\Omega}^{a} \mu(P)=\int_{C_{n}(\Omega)} G_{\Omega}^{a}(P, Q) d \mu(Q)=\sum_{k=0}^{\infty} \widehat{R}_{M_{\Omega}^{a}(\cdot, \infty)}^{E_{k}}(P)
$$

is a finite-valued superfunction on $C_{n}(\Omega)$ and

$$
G_{\Omega}^{a} \mu(P) \geq \int_{C_{n}(\Omega)} G_{\Omega}^{a}(P, Q) d \lambda_{E_{k}}^{a}(Q)=\widehat{R}_{M_{\Omega}^{a}(\cdot, \infty)}^{E_{k}}(P)=V(r) \varphi(\Theta)
$$

for any $P=(r, \Theta) \in B_{E_{k}}$, and from (1.16) or (1.17)

$$
G_{\Omega}^{a} \mu(P) \geq C^{\prime} V(r) \varphi(\Theta)
$$

for any $P=(r, \Theta) \in C_{n}(\Omega ;(0,1])$, where

$$
C^{\prime}=C_{1} \int_{C_{n}(\Omega ;[5 / 4, \infty))} W(t) \varphi(\Phi) d \mu(t, \Phi)
$$

If we set

$$
E^{\prime}=\bigcup_{k=0}^{\infty} B_{E_{k}}, \quad E_{1}=E \cap C_{n}(\Omega ;(0,1]), \quad C=\min \left(C^{\prime}, 1\right),
$$

then

$$
E^{\prime} \subset\left\{P=(r, \Theta) \in C_{n}(\Omega) ; G_{\Omega}^{a} \mu(P) \geq C V(r) \varphi(\Theta)\right\} .
$$


Hence by Lemma 3.5, $E^{\prime}$ is $a$-minimally thin at $\infty$ with respect to $C_{n}(\Omega)$; namely, there is a point $P^{\prime} \in C_{n}(\Omega)$ such that

$$
\widehat{R}_{M_{\Omega}^{a}(\cdot, \infty)}^{E^{\prime}}\left(P^{\prime}\right) \neq M_{\Omega}^{a}\left(P^{\prime}, \infty\right)
$$

Since $E^{\prime}$ is equal to $E$ except an $a$-polar set, we know that

$$
\widehat{R}_{M_{\Omega}^{a}(\cdot, \infty)}^{E^{\prime}}(P)=\widehat{R}_{M_{\Omega}^{a}(\cdot, \infty)}^{E}(P)
$$

for any $P \in C_{n}(\Omega)$, and hence

$$
\widehat{R}_{M_{\Omega}^{a}(\cdot, \infty)}^{E}\left(P^{\prime}\right) \neq M_{\Omega}^{a}\left(P^{\prime}, \infty\right)
$$

So $E$ is $a$-minimally thin at $\infty$ with respect to $C_{n}(\Omega)$.

Proof of Theorem 2.6. Let a subset $E$ of $C_{n}(\Omega)$ be an $a$-rarefied set at $\infty$ with respect to $C_{n}(\Omega)$. Then there exists a positive superfunction $v(P)$ on $C_{n}(\Omega)$ such that $c(v, a) \equiv 0$ and

$$
E \subset H_{v}
$$

By Lemma 3.6 we can find two positive measures $\mu$ on $C_{n}(\Omega)$ and $v$ on $S_{n}(\Omega)$ such that

$$
\begin{aligned}
v(P)= & c_{O}(v, a) M_{\Omega}^{a}(P, O)+\int_{C_{n}(\Omega)} G_{\Omega}^{a}(P, Q) d \mu(Q) \\
& +\int_{S_{n}(\Omega)} \frac{\partial G_{\Omega}^{a}(P, Q)}{\partial n_{Q}} d v(Q) \quad\left(P \in C_{n}(\Omega)\right) .
\end{aligned}
$$

Set

$$
v(P)=c_{O}(v, a) M_{\Omega}^{a}(P, O)+B_{1}^{(k)}(P)+B_{2}^{(k)}(P)+B_{3}^{(k)}(P),
$$

where

$$
\begin{aligned}
B_{1}^{(k)}(P)= & \int_{C_{n}\left(\Omega ;\left(0,2^{k-1}\right]\right)} G_{\Omega}^{a}(P, Q) d \mu(Q)+\int_{S_{n}\left(\Omega ;\left(0,2^{k-1}\right]\right)} \frac{\partial G_{\Omega}^{a}(P, Q)}{\partial n_{Q}} d v(Q), B_{2}^{(k)}(P) \\
= & \int_{C_{n}\left(\Omega ;\left(2^{k-1}, 2^{k+2}\right)\right)} G_{\Omega}^{a}(P, Q) d \mu(Q)+\int_{S_{n}\left(\Omega ;\left(2^{k-1}, 2^{k+2}\right)\right)} \frac{\partial G_{\Omega}^{a}(P, Q)}{\partial n_{Q}} d v(Q), B_{3}^{(k)}(P) \\
= & \int_{C_{n}\left(\Omega ;\left[2^{k+2}, \infty\right)\right)} G_{\Omega}^{a}(P, Q) d \mu(Q) \\
& +\int_{S_{n}\left(\Omega ;\left[2^{k+2}, \infty\right)\right)} \frac{\partial G_{\Omega}^{a}(P, Q)}{\partial n_{Q}} d v(Q) \quad\left(P \in C_{n}(\Omega) ; k=1,2,3, \ldots\right) .
\end{aligned}
$$


First we will prove there exists an integer $N$ such that

$$
H_{v} \cap I_{k}(\Omega) \subset\left\{P=(r, \Theta) \in I_{k}(\Omega) ; B_{2}^{(k)}(P) \geq \frac{1}{2} V(r)\right\}
$$

for any integer $k \geq N$. Since $v(P)$ is finite almost everywhere on $C_{n}(\Omega)$, we may apply Lemmas 3.3 and 3.4 to

$$
\int_{C_{n}(\Omega)} G_{\Omega}^{a}(P, Q) d \mu(Q), \quad \int_{S_{n}(\Omega)} \frac{\partial G_{\Omega}^{a}(P, Q)}{\partial n_{Q}} d v(Q),
$$

respectively; then we can take an integer $N$ such that

$$
\begin{gathered}
\frac{W\left(2^{k-1}\right)}{V\left(2^{k-1}\right)} \int_{C_{n}\left(\Omega ;\left(0,2^{k-1}\right]\right)} V(t) \varphi(\Phi) d \mu(t, \Phi) \leq \frac{1}{12 J_{\Omega} C_{2}}, \\
\int_{\left.C_{n}\left(\Omega ; 2^{k+2}, \infty\right)\right)} W(t) \varphi(\Phi) d \mu(t, \Phi) \leq \frac{1}{12 J_{\Omega} C_{2}}, \\
\frac{W\left(2^{k-1}\right)}{V\left(2^{k-1}\right)} \int_{S_{n}\left(\Omega ;\left(0,2^{k-1}\right]\right)} V(t) t^{-1} \frac{\partial \varphi(\Phi)}{\partial n_{\Phi}} d v(t, \Phi) \leq \frac{1}{12 J_{\Omega} C_{2}}, \\
\int_{\left.S_{n}\left(\Omega ; 2^{k+2}, \infty\right)\right)} W(t) t^{-1} \frac{\partial \varphi(\Phi)}{\partial n_{\Phi}} d v(t, \Phi) \leq \frac{1}{12 J_{\Omega} C_{2}}
\end{gathered}
$$

for any integer $k \geq N$, where

$$
J_{\Omega}=\sup _{\Theta \in \Omega} \varphi(\Theta)
$$

Then for any $P=(r, \Theta) \in I_{k}(\Omega)(k \geq N)$, we have

$$
\begin{aligned}
B_{1}^{(k)}(P) \leq & C_{2} J_{\Omega} W(r) \int_{C_{n}\left(\Omega ;\left(0,2^{k-1}\right]\right)} V(t) \varphi(\Phi) d \mu(t, \Phi) \\
& +C_{2} J_{\Omega} W(r) \int_{S_{n}\left(\Omega ;\left(0,2^{k-1}\right]\right)} V(t) t^{-1} \frac{\partial \varphi(\Phi)}{\partial n_{\Phi}} d v(t, \Phi) \leq \frac{V(r)}{6}
\end{aligned}
$$

from (1.16) or (1.17), (3.3) or (3.4), (4.50), and (4.52), and

$$
\begin{aligned}
B_{3}^{(k)}(P) \leq & C_{2} J_{\Omega} V(r) \int_{C_{n}\left(\Omega ;\left[2^{k+2}, \infty\right)\right)} W(t) \varphi(\Phi) d \mu(t, \Phi) \\
& +C_{2} J_{\Omega} V(r) \int_{S_{n}\left(\Omega ;\left[2^{k+2}, \infty\right)\right)} W(t) t^{-1} \frac{\partial \varphi(\Phi)}{\partial n_{\Phi}} d v(t, \Phi) \leq \frac{V(r)}{6}
\end{aligned}
$$


from (1.16) or (1.17), (3.3) or (3.4), (4.51), and (4.53). Further we can assume that

$$
6 \kappa c_{O}(v, a) J_{\Omega} \leq V(r) W(r)^{-1}
$$

for any $P=(r, \Theta) \in I_{k}(\Omega)(k \geq N)$. Hence if $P=(r, \Theta) \in I_{k}(\Omega) \cap H_{v}(k \geq N)$, we obtain

$$
B_{2}^{(k)}(P) \geq v(P)-\frac{V(r)}{6}-B_{1}^{(k)}(P)-B_{3}^{(k)}(P) \geq \frac{V(r)}{2}
$$

from (4.46) which gives (4.48).

We see from (4.44) and (4.48) that

$$
B_{2}^{(k)}(P) \geq \frac{1}{2} V\left(2^{k}\right) \quad(k \geq N)
$$

for any $P \in E_{k}$. Define a function $u_{k}(P)$ on $C_{n}(\Omega)$ by

$$
u_{k}(P)=2 V\left(2^{k}\right)^{-1} B_{2}^{(k)}(P) .
$$

Then

$$
\begin{gathered}
u_{k}(P) \geq 1 \quad\left(P \in E_{k}, k \geq N\right), \\
u_{k}(P)=\int_{C_{n}(\Omega)} G_{\Omega}^{a}(P, Q) d \mu_{k}(Q)+\int_{S_{n}(\Omega)} \frac{\partial G_{\Omega}^{a}(P, Q)}{\partial n_{Q}} d v_{k}(Q)
\end{gathered}
$$

with two measures

$$
\begin{aligned}
d \mu_{k}(Q) & = \begin{cases}2 V\left(2^{k}\right)^{-1} d \mu(Q) & \left(Q \in C_{n}\left(\Omega ;\left(2^{k-1}, 2^{k+2}\right)\right)\right), \\
0 & \left(Q \in C_{n}\left(\Omega ;\left(0,2^{k-1}\right]\right) \cup C_{n}\left(\Omega ;\left[2^{k+2}, \infty\right)\right)\right),\end{cases} \\
d v_{k}(Q) & = \begin{cases}2 V\left(2^{k}\right)^{-1} d v(Q) & \left(Q \in S_{n}\left(\Omega ;\left(2^{k-1}, 2^{k+2}\right)\right)\right), \\
0 & \left(Q \in S_{n}\left(\Omega ;\left(0,2^{k-1}\right]\right) \cup S_{n}\left(\Omega ;\left[2^{k+2}, \infty\right)\right)\right) .\end{cases}
\end{aligned}
$$

Hence by applying Lemma 3.7 to $u_{k}(P)$, we obtain

$$
\begin{aligned}
\lambda_{\Omega}^{a}\left(E_{k}\right) \leq & 2 V\left(2^{k}\right)^{-1} \int_{C_{n}\left(\Omega ;\left(2^{k-1}, 2^{k+2}\right)\right)} V(t) \varphi(\Phi) d \mu(t, \Phi) \\
& +2 V\left(2^{k}\right)^{-1} \int_{S_{n}\left(\Omega ;\left(2^{k-1}, 2^{k+2}\right)\right)} V(t) t^{-1} \frac{\partial \varphi(\Phi)}{\partial n_{\Phi}} d v(t, \Phi) \quad(k \geq N) .
\end{aligned}
$$


Finally we have by (1.11), (1.12), and (1.14)

$$
\sum_{k=N}^{\infty} W\left(2^{k}\right) \lambda_{\Omega}^{a}\left(E_{k}\right) \lesssim \int_{C_{n}\left(\Omega ;\left(2^{N-1}, \infty\right)\right)} W(t) \varphi(\Phi) d \mu(t, \Phi)+\int_{S_{n}\left(\Omega ;\left(2^{N-1}, \infty\right)\right)} W(t) t^{-1} \frac{\partial \varphi(\Phi)}{\partial n_{\Phi}} d v(t, \Phi)
$$

If we take a sufficiently large $N$, then the integrals of the right side are finite from Lemmas 3.3 and 3.4 .

Suppose that a subset $E$ of $C_{n}(\Omega)$ satisfies

$$
\sum_{k=0}^{\infty} W\left(2^{k}\right) \lambda_{\Omega}^{a}\left(E_{k}\right)<\infty
$$

Then we apply the second part of Lemma 3.7 to $E_{k}$ and get

$$
\sum_{k=1}^{\infty} W\left(2^{k}\right)\left\{\int_{C_{n}(\Omega)} V(t) \varphi(\Phi) d \mu_{k}^{*}(t, \Phi)+\int_{S_{n}(\Omega)} V(t) t^{-1} \frac{\partial \varphi(\Phi)}{\partial n_{\Phi}} d v_{k}^{*}(t, \Phi)\right\}<\infty,
$$

where $\mu_{k}^{*}$ and $v_{k}^{*}$ are two positive measures on $C_{n}(\Omega)$ and $S_{n}(\Omega)$, respectively, such that

$$
\widehat{R}_{1}^{E_{k}}(P)=\int_{C_{n}(\Omega)} G_{\Omega}^{a}(P, Q) d \mu_{k}^{*}(Q)+\int_{S_{n}(\Omega)} \frac{\partial G_{\Omega}^{a}(P, Q)}{\partial n_{Q}} d v_{k}^{*}(Q) .
$$

Consider a function $v_{0}(P)$ on $C_{n}(\Omega)$ defined by

$$
v_{0}(P)=\sum_{k=-1}^{\infty} V\left(2^{k+1}\right) \hat{R}_{1}^{E_{k}}(P) \quad\left(P \in C_{n}(\Omega)\right)
$$

where

$$
E_{-1}=E \cap\left\{P=(r, \Theta) \in C_{n}(\Omega) ; 0<r<1\right\} .
$$

Then $v_{0}(P)$ is a superfunction or identically $\infty$ on $C_{n}(\Omega)$. We take any positive integer $k_{0}$ and represent $v_{0}(P)$ by

$$
v_{0}(P)=v_{1}(P)+v_{2}(P)
$$

where

$$
v_{1}(P)=\sum_{k=-1}^{k_{0}+1} V\left(2^{k+1}\right) \hat{R}_{1}^{E_{k}}(P), \quad v_{2}(P)=\sum_{k=k_{0}+2}^{\infty} V\left(2^{k+1}\right) \hat{R}_{1}^{E_{k}}(P) .
$$


Since $\mu_{k}^{*}$ and $v_{k}^{*}$ are concentrated on $B_{E_{k}} \subset \overline{E_{k}} \cap C_{n}(\Omega)$ and $B_{E_{k}}^{\prime} \subset \overline{E_{k}} \cap S_{n}(\Omega)$, respectively, we have from (1.16) or (1.17), (3.3) or (3.4), (1.11), and (1.12) that

$$
\begin{aligned}
\int_{C_{n}(\Omega)} G_{\Omega}^{a}\left(P^{\prime}, Q\right) d \mu_{k}^{*}(Q) \leq & C_{2} V\left(r^{\prime}\right) \varphi\left(\Theta^{\prime}\right) \int_{C_{n}(\Omega)} W(t) \varphi(\Phi) d \mu_{k}^{*}(t, \Phi) \\
\leq & C_{2} W\left(2^{k}\right) V\left(2^{k}\right)^{-1} V\left(r^{\prime}\right) \varphi\left(\Theta^{\prime}\right) \\
& \times \int_{C_{n}(\Omega)} V(t) \varphi(\Phi) d \mu_{k}^{*}(t, \Phi), \int_{S_{n}(\Omega)} \frac{\partial G_{\Omega}^{a}\left(P^{\prime}, Q\right)}{\partial n_{Q}} d v_{k}^{*}(Q) \\
\leq & C_{2} W\left(2^{k}\right) V\left(2^{k}\right)^{-1} V\left(r^{\prime}\right) \varphi\left(\Theta^{\prime}\right) \int_{S_{n}(\Omega)} V(t) t^{-1} \frac{\partial \varphi(\Phi)}{\partial n_{\Phi}} d v_{k}^{*}(t, \Phi)
\end{aligned}
$$

for a point $P^{\prime}=\left(r^{\prime}, \Theta^{\prime}\right) \in C_{n}(\Omega)$, where $r^{\prime} \leq 2^{k_{0}+1}$ and $k \leq k_{0}+2$. Hence we know by (1.11), (1.12), and (1.14) that

$$
\begin{aligned}
v_{2}\left(P^{\prime}\right) \lesssim & V\left(r^{\prime}\right) \varphi\left(\Theta^{\prime}\right) \sum_{k=k_{0}+2}^{\infty} W\left(2^{k}\right) \int_{C_{n}(\Omega)} V(t) \varphi(\Phi) d \mu_{k}^{*}(t, \Phi) \\
& +V\left(r^{\prime}\right) \varphi\left(\Theta^{\prime}\right) \sum_{k=k_{0}+2}^{\infty} W\left(2^{k}\right) \int_{S_{n}(\Omega)} V(t) t^{-1} \frac{\partial \varphi(\Phi)}{\partial n_{\Phi}} d v_{k}^{*}(t, \Phi) .
\end{aligned}
$$

This and (4.66) show that $v_{2}\left(P^{\prime}\right)$ is finite, and hence $v_{0}(P)$ is a positive superfunction on $C_{n}(\Omega)$. To see

$$
c\left(v_{0}, a\right)=\inf _{P \in C_{n}(\Omega)} \frac{v_{0}(P)}{M_{\Omega}^{a}(P, \infty)}=0,
$$

we consider the representations of $v_{0}(P), v_{1}(P)$, and $v_{2}(P)$ by Lemma 3.6 as follows:

$$
\begin{aligned}
v_{0}(P)= & c\left(v_{0}, a\right) M_{\Omega}^{a}(P, \infty)+c_{O}\left(v_{0}, a\right) M_{\Omega}^{a}(P, O) \\
& +\int_{C_{n}(\Omega)} G_{\Omega}^{a}(P, Q) d \mu_{(0)}(Q)+\int_{S_{n}(\Omega)} \frac{\partial G_{\Omega}^{a}(P, Q)}{\partial n_{Q}} d v_{(0)}(Q), v_{1}(P) \\
= & c\left(v_{1}, a\right) M_{\Omega}^{a}(P, \infty)+c_{O}\left(v_{1}, a\right) M_{\Omega}^{a}(P, O) \\
& +\int_{C_{n}(\Omega)} G_{\Omega}^{a}(P, Q) d \mu_{(1)}(Q)+\int_{S_{n}(\Omega)} \frac{\partial G_{\Omega}^{a}(P, Q)}{\partial n_{Q}} d v_{(1)}(Q), v_{2}(P) \\
= & c\left(v_{2}, a\right) M_{\Omega}^{a}(P, \infty)+c_{O}\left(v_{2}, a\right) M_{\Omega}^{a}(P, O) \\
& +\int_{C_{n}(\Omega)} G_{\Omega}^{a}(P, Q) d \mu_{(2)}(Q)+\int_{S_{n}(\Omega)} \frac{\partial G_{\Omega}^{a}(P, Q)}{\partial n_{Q}} d v_{(2)}(Q) .
\end{aligned}
$$


It is evident from $(4.67)$ that $c\left(v_{1}, a\right)=0$ for any $k_{0}$. Since $c\left(v_{0}, a\right)=c\left(v_{2}, a\right)$ and

$$
\begin{aligned}
c\left(v_{2}, a\right)= & \inf _{P \in C_{n}(\Omega)} \frac{v_{2}(P)}{M_{\Omega}^{a}(P, \infty)} \leq \frac{v_{2}\left(P^{\prime}\right)}{M_{\Omega}^{a}\left(P^{\prime}, \infty\right)} \lesssim \sum_{k=k_{0}+2}^{\infty} W\left(2^{k}\right) \int_{C_{n}(\Omega)} V(t) \varphi(\Phi) d \mu_{k}^{*}(t, \Phi) \\
& +\sum_{k=k_{0}+2}^{\infty} W\left(2^{k}\right) \int_{S_{n}(\Omega)} V(t) t^{-1} \frac{\partial \varphi(\Phi)}{\partial n_{\Phi}} d v_{k}^{*}(t, \Phi) \longrightarrow 0 \quad\left(k_{0} \longrightarrow \infty\right)
\end{aligned}
$$

from (4.66) and (4.73), we know $c\left(v_{0}, a\right)=0$ which is (4.74). Since $\widehat{R}_{1}^{E_{k}}=1$ on $B_{E_{k}} \subset \overline{E_{k}} \cap$ $C_{n}(\Omega)$, we know that

$$
v_{0}(P) \geq V\left(2^{k+1}\right) \geq V(r)
$$

for any $P=(r, \Theta) \in B_{E_{k}}(k=-1,0,1,2, \ldots)$. We set $E^{\prime}=\cup_{k=-1}^{\infty} B_{E_{k}}$; then

$$
E^{\prime} \subset H_{v_{0}}
$$

Since $E^{\prime}$ is equal to $E$ except an $a$-polar set $S$, we can take another positive superfunction $v_{3}$ on $C_{n}(\Omega)$ such that $v_{3}=G_{\Omega}^{a} \eta$ with a positive measure $\eta$ on $C_{n}(\Omega)$, and $v_{3}$ is identically $\infty$ on $S$. Define a positive superfunction $v$ on $C_{n}(\Omega)$ by

$$
v=v_{0}+v_{3} .
$$

Since $c\left(v_{3}, a\right)=0$, it is easy to see from (4.74) that $c(v, a)=0$. In addition, we know from (4.78) that $E \subset H_{v}$. Then the subset $E$ of $C_{n}(\Omega)$ is $a$-rarefied at $\infty$ with respect to $C_{n}(\Omega)$.

Proof of Theorem 2.8. By Lemma 3.6 we have

$$
\begin{aligned}
v(P)= & c(v, a) M_{\Omega}^{a}(P, \infty)+c_{O}(v, a) M_{\Omega}^{a}(P, O)+\int_{C_{n}(\Omega)} G_{\Omega}^{a}(P, Q) d \mu(Q) \\
& +\int_{S_{n}(\Omega)} \frac{\partial G_{\Omega}^{a}(P, Q)}{\partial n_{Q}} d v(Q)
\end{aligned}
$$

for a unique positive measure $\mu$ on $C_{n}(\Omega)$ and a unique positive measure $v$ on $S_{n}(\Omega)$, respectively; then

$$
v_{1}(P)=v(P)-c(v, a) M_{\Omega}^{a}(P, \infty)-c_{O}(v, a) M_{\Omega}^{a}(P, O) \quad\left(P=(r, \Theta) \in C_{n}(\Omega)\right)
$$

also is a positive superfunction on $C_{n}(\Omega)$ such that

$$
\inf _{P=(r, \Theta) \in C_{n}(\Omega)} \frac{v_{1}(P)}{M_{\Omega}^{a}(P, \infty)}=0 .
$$


Next we will prove there exists an $a$-rarefied set $E$ at $\infty$ with respect to $C_{n}(\Omega)$ such that

$$
v_{1}(P) V(r)^{-1} \quad\left(P=(r, \Theta) \in C_{n}(\Omega)\right)
$$

uniformly converges to 0 on $C_{n}(\Omega) \backslash E$ as $r \rightarrow \infty$. Let $\left\{\varepsilon_{i}\right\}$ be a sequence of positive numbers $\varepsilon_{i}$ satisfying $\varepsilon_{i} \rightarrow 0$ as $i \rightarrow \infty$, and put

$$
E_{i}=\left\{P=(r, \Theta) \in C_{n}(\Omega) ; v_{1}(P) \geq \varepsilon_{i} V(r)\right\} \quad(k=1,2,3, \ldots)
$$

Then $E_{i}(k=1,2,3, \ldots)$ are $a$-rarefied sets at $\infty$ with respect to $C_{n}(\Omega)$, and hence by Theorem 2.6

$$
\sum_{k=0}^{\infty} W\left(2^{k}\right) \lambda_{\Omega}^{a}\left(\left(E_{i}\right)_{k}\right)<\infty \quad(i=1,2,3, \ldots)
$$

We take a sequence $\left\{q_{i}\right\}$ such that

$$
\sum_{k=q_{i}}^{\infty} W\left(2^{k}\right) \lambda_{\Omega}^{a}\left(\left(E_{i}\right)_{k}\right)<\frac{1}{2^{i}} \quad(i=1,2,3, \ldots)
$$

and set

$$
E=\cup_{i=1}^{\infty} \cup_{k=q_{i}}^{\infty}\left(E_{i}\right)_{k}
$$

Because $\lambda_{\Omega}^{a}$ is a countably subadditive set function as in Aikawa [25], Essén, and Jackson [4],

$$
\lambda_{\Omega}^{a}\left(E_{m}\right) \leq \sum_{i=1}^{\infty} \sum_{k=q_{i}}^{\infty} \lambda_{\Omega}^{a}\left(E_{i} \cap I_{k} \cap I_{m}\right) \quad(m=1,2,3, \ldots)
$$

Since

$$
\sum_{m=1}^{\infty} \lambda_{\Omega}^{a}\left(E_{m}\right) W\left(2^{m}\right) \leq \sum_{i=1}^{\infty} \sum_{k=q_{i}}^{\infty} \sum_{m=1}^{\infty} \lambda_{\Omega}^{a}\left(E_{i} \cap I_{k} \cap I_{m}\right) W\left(2^{m}\right)=\sum_{i=1}^{\infty} \sum_{k=q_{i}}^{\infty} \lambda_{\Omega}^{a}\left(\left(E_{i}\right)_{k}\right) W\left(2^{k}\right) \leq \sum_{i=1}^{\infty} \frac{1}{2^{i}}=1,
$$

by Theorem 2.6 we know that $E$ is an $a$-rarefied set at $\infty$ with respect to $C_{n}(\Omega)$. It is easy to see that

$$
v(P) V(r)^{-1} \quad\left(P=(r, \Theta) \in C_{n}(\Omega)\right)
$$

uniformly converges to 0 on $C_{n}(\Omega) \backslash E$ as $r \rightarrow \infty$. 
Proof of Theorem 2.10. Since $\lambda_{E_{k}}^{a}$ is concentrated on $B_{E_{k}} \subset \overline{E_{k}} \cap C_{n}(\Omega)$, we see that

$$
\gamma_{\Omega}^{a}\left(E_{k}\right)=\int_{C_{n}(\Omega)} \widehat{R}_{M_{\Omega}^{a}(\cdot, \infty)}^{E_{k}}(P) d \lambda_{E_{k}}^{a}(P) \leq \int_{C_{n}(\Omega)} M_{\Omega}^{a}(P, \infty) d \lambda_{E_{k}}^{a}(P) \leq J_{\Omega} V\left(2^{k+1}\right) \lambda_{\Omega}^{a}\left(E_{k}\right),
$$

and hence

$$
\sum_{k=0}^{\infty} r_{\Omega}^{a}\left(E_{k}\right) W\left(2^{k}\right) V\left(2^{k}\right)^{-1} \lesssim \sum_{k=0}^{\infty} W\left(2^{k}\right) \lambda_{\Omega}^{a}\left(E_{k}\right)
$$

which gives the conclusion of the first part with Theorems 2.5 and 2.6. To prove the second part, we put $J_{\Omega}^{\prime}=\min _{\Theta \in \overline{\Omega^{\prime}}} \varphi(\Theta)$. Since

$$
\begin{gathered}
M_{\Omega}^{a}(\cdot, \infty)=V(r) \varphi(\Theta) \geq J_{\Omega}^{\prime} V(r) \geq J_{\Omega}^{\prime} V\left(2^{k}\right) \quad\left(P=(r, \Theta) \in E_{k}\right), \\
\widehat{R}_{M_{\Omega}^{a}(\cdot, \infty)}^{E_{k}}(P)=M_{\Omega}^{a}(\cdot, \infty)
\end{gathered}
$$

for any $P=(r, \Theta) \in B_{E_{k}}$, we have

$$
\gamma_{\Omega}^{a}\left(E_{k}\right)=\int_{C_{n}(\Omega)} \widehat{R}_{M_{\Omega}^{a}(\cdot, \infty)}^{E_{k}}(P) d \lambda_{E_{k}}^{a}(P) \geq J_{\Omega}^{\prime} V\left(2^{k}\right) \lambda_{\Omega}^{a}\left(E_{k}\right)
$$

Since

$$
J_{\Omega}^{\prime} \sum_{k=0}^{\infty} \lambda_{\Omega}^{a}\left(E_{k}\right) W\left(2^{k}\right) \leq \sum_{k=0}^{\infty} V\left(2^{k}\right)^{-1} W\left(2^{k}\right) r_{\Omega}^{a}\left(E_{k}\right)<\infty
$$

from Theorem 2.5, it follows from Theorem 2.6 that $E$ is $a$-rarefied at $\infty$ with respect to $C_{n}(\Omega)$.

\section{Acknowledgment}

The authors wish to express their appreciation to the referee for her or his careful reading and some useful suggestions which led to an improvement of their original paper. The work is supported by SRFDP (No. 20100003110004) and NSF of China (No. 10671022 and No. 11101039).

\section{References}

[1] J. Lelong-Ferrand, "Étude au voisinage de la frontière des fonctions subharmoniques positives dans un demi-espace," Annales Scientifiques de l'École Normale Supérieure, vol. 66, pp. 125-159, 1949.

[2] L. Naïm, "Sur le rôle de la frontière de R. S. Martin dans la théorie du potentiel," Annales de l'Institut Fourier, vol. 7, pp. 183-281, 1957.

[3] D. H. Armitage and S. J. Gardiner, Classical Potential Theory, Springer Monographs in Mathematics, Springer, London, UK, 2001. 
[4] M. Essén and H. L. Jackson, "On the covering properties of certain exceptional sets in a half-space," Hiroshima Mathematical Journal, vol. 10, no. 2, pp. 233-262, 1980.

[5] I. Miyamoto and H. Yoshida, "Two criterions of Wiener type for minimally thin sets and rarefied sets in a cone," Journal of the Mathematical Society of Japan, vol. 54, no. 3, pp. 487-512, 2002.

[6] V.S. Azarin, "Generalization of a theorem of Hayman on subharmonic functions in an $m$-dimensional cone," American Mathematical Society Translations: Series 2, vol. 80, pp. 119-138, 1969.

[7] M. Reed and B. Simon, Methods of Modern Mathematical Physics, vol. 3, Academic Press, New York, NY, USA, 1970.

[8] M. Cranston, "Conditional Brownian motion, Whitney squares and the conditional gauge theorem," in Seminar on Stochastic Processes, 1988 (Gainesville, FL, 1988), vol. 17, pp. 109-119, Birkhäuser, Boston, Mass, USA, 1989.

[9] M. Cranston, E. Fabes, and Z. Zhao, "Conditional gauge and potential theory for the Schrödinger operator," Transactions of the American Mathematical Society, vol. 307, no. 1, pp. 174-194, 1988.

[10] L. Qiao and G. Deng, "A theorem of Phragmén-Lindelöf type for subfunctions in a cone," Glasgow Mathematical Journal, vol. 53, no. 3, pp. 599-610, 2011.

[11] A. Escassut, W. Tutschke, and C. C. Yang, Some Topics on Value Distribution and Differentility in Complex and P-Adic Analysis, Science Press, Beijng, China, 2008.

[12] A. I. Kheyfits, "Dirichlet problem for the Schrödinger operator in a half-space with boundary data of arbitrary growth at infinity," Differential and Integral Equations, vol. 10, no. 1, pp. 153-164, 1997.

[13] B. Levin and A. Kheyfits, "Asymptotic behavior of subfunctions of the stationary Schrödinger operator," http://arxiv.org/abs/math/0211328v1.

[14] G. Rosenblum, M. Solomyak, and M. Shubin, Spectral Theory of Differential Operators, VINITI, Moscow, Russia, 1989.

[15] D. Gilbarg and N. S. Trudinger, Elliptic Partial Differential Equations of Second Order, Springer, Berlin, Germany, 1977.

[16] B. Simon, "Schrödinger semigroups," Bulletin American Mathematical Society, vol. 7, no. 3, pp. 447-526, 1982.

[17] G. M. Verzhbinskii and V. G. Maz'ya, “Asymptotic behavior of solutions of elliptic equations of the second order close to a boundary. I," Siberian Mathematical Journal, vol. 12, no. 6, pp. 874-899, 1971.

[18] M. Essén and J. L. Lewis, "The generalized Ahlfors-Heins theorem in certain $d$-dimensional cones," Mathematica Scandinavica, vol. 33, pp. 113-129, 1973.

[19] M. Brelot, On Topologies and Boundaries in Potential Theory, vol. 175 of Lecture Notes in Mathematics, Springer, Berlin, Germany, 1971.

[20] R. S. Martin, "Minimal positive harmonic functions," Transactions of the American Mathematical Society, vol. 49, pp. 137-172, 1941.

[21] J. L. Doob, Classical Potential Theory and Its Probabilistic Counterpart, Springer, London, UK, 1984.

[22] H. Aikawa and M. Essén, Potential Theory-Selected Topics, vol. 1633 of Lecture Notes in Mathematics, Springer, Berlin, Germany, 1996.

[23] H. Yoshida, "Nevanlinna norm of a subharmonic function on a cone or on a cylinder," Proceedings of the London Mathematical Society: Series 3, vol. 54, no. 2, pp. 267-299, 1987.

[24] L. Qiao, Some researches on generalized harmonic and superharmonic functions [Dissertation of Beijing Normal University], Beijing Normal University, Beijng, China, 2010.

[25] H. Aikawa, "On the behavior at infinity of nonnegative superharmonic functions in a half space," Hiroshima Mathematical Journal, vol. 11, no. 2, pp. 425-441, 1981. 


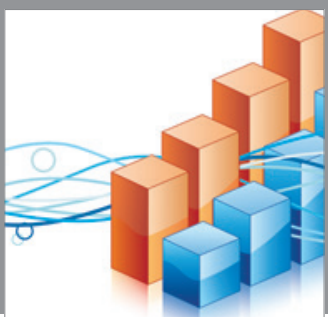

Advances in

Operations Research

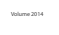

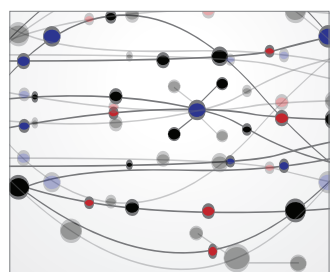

\section{The Scientific} World Journal
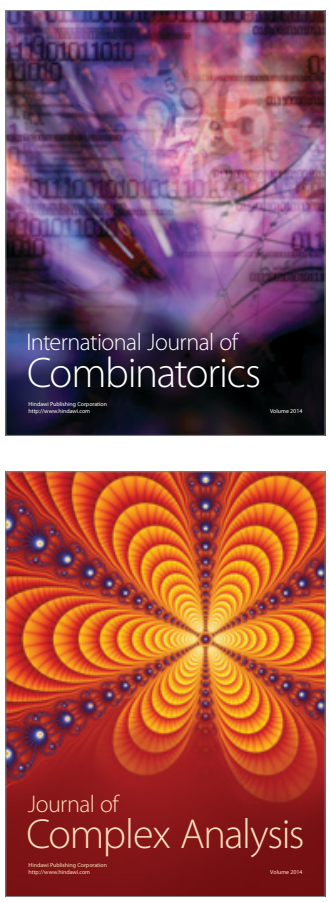

International Journal of

Mathematics and

Mathematical

Sciences
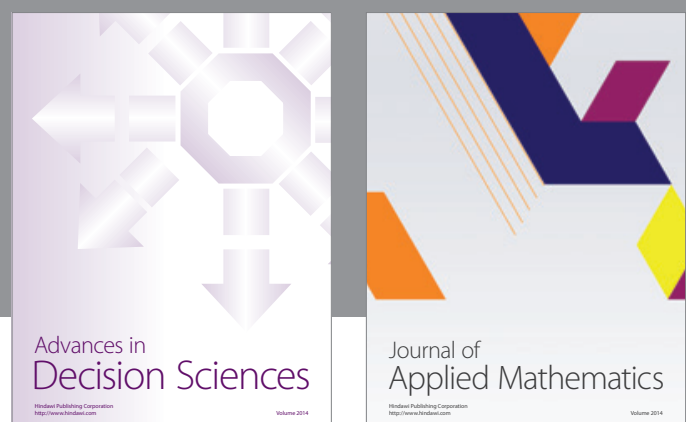

Journal of

Applied Mathematics
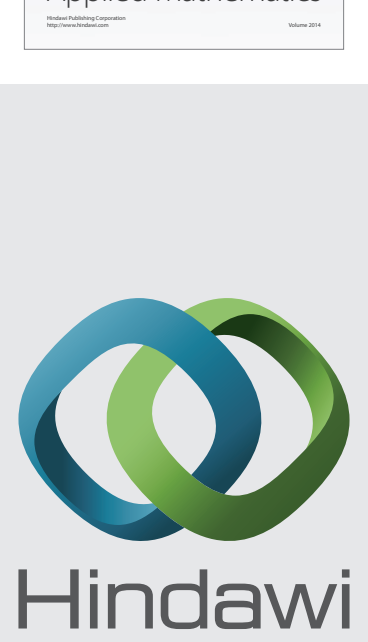

Submit your manuscripts at http://www.hindawi.com
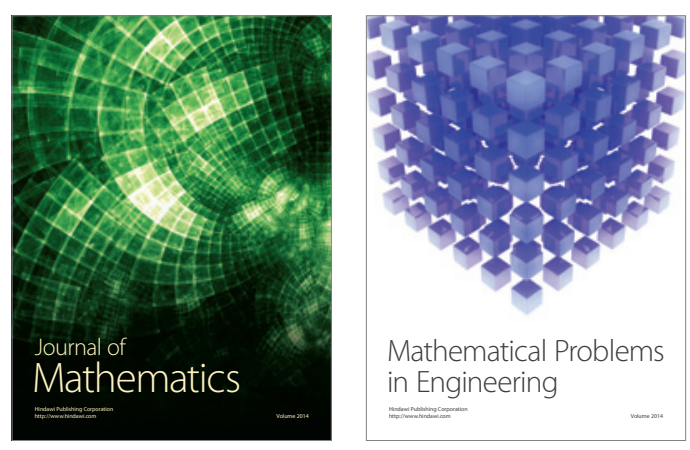

Mathematical Problems in Engineering
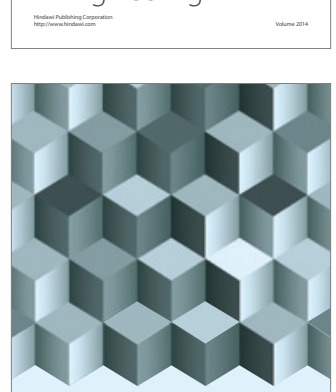

Journal of

Function Spaces
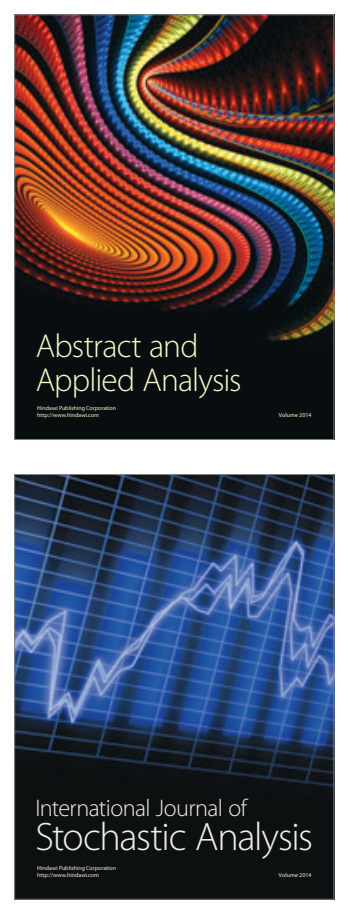

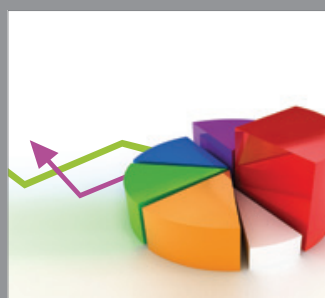

ournal of

Probability and Statistics

Promensencen
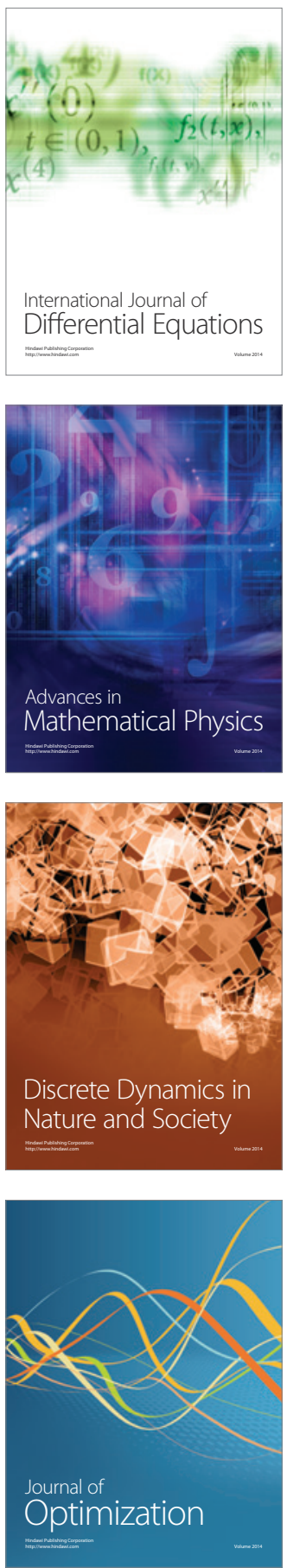